\title{
Strong Equivalence of Qualitative Optimization Problems
}

\author{
Wolfgang Faber \\ Department of Mathematics \\ University of Calabria \\ Via P. Bucci cubo 30B, 87036 Rende, Italy \\ Mirosław Truszczyński \\ Department of Computer Science \\ University of Kentucky \\ 329 Rose Street, Lexington, KY 40506-00633, USA
}

\section{Stefan Woltran}

Institute of Information Systems

Vienna University of Technology

Favoritenstraße 9-11, 1040 Vienna, Austria
FABER@MAT.UNICAL.IT

MIREK@CS.UKY.EDU

WOLTRAN@DBAI.TUWIEN.AC.AT

\begin{abstract}
We introduce the framework of qualitative optimization problems (or, simply, optimization problems) to represent preference theories. The formalism uses separate modules to describe the space of outcomes to be compared (the generator) and the preferences on outcomes (the selector). We consider two types of optimization problems. They differ in the way the generator, which we model by a propositional theory, is interpreted: by the standard propositional logic semantics, and by the equilibrium-model (answer-set) semantics. Under the latter interpretation of generators, optimization problems directly generalize answer-set optimization programs proposed previously. We study strong equivalence of optimization problems, which guarantees their interchangeability within any larger context. We characterize several versions of strong equivalence obtained by restricting the class of optimization problems that can be used as extensions and establish the complexity of associated reasoning tasks. Understanding strong equivalence is essential for modular representation of optimization problems and rewriting techniques to simplify them without changing their inherent properties.
\end{abstract}

\section{Introduction}

We introduce the framework of qualitative optimization problems in which, following the design of answer-set optimization (ASO) programs (Brewka, Niemelä, \& Truszczyński, 2003), we use separate modules to describe the space of outcomes to be compared (the generator) and the preferences on the outcomes (the selector). In all optimization problems we consider, the selector module follows the syntax and the semantics of preference modules in ASO programs, and the generator is given by a propositional theory. When this propositional theory is interpreted according to the standard propositional logic semantics, that is, outcomes to be compared are classical models of the generator, we speak about classical optimization problems (CO problems, for short). When the generator theory is interpreted by the semantics of equilibrium models (Pearce, 1997), we speak about answer-set optimization problems (ASO problems, for short). We use this terminology, as equilibrium models are 
usually referred to as answer sets (Ferraris, 2005) for historical reasons. In ASO problems, the answer sets of the generator are the outcomes used to determine the optimal outcomes.

Representing and reasoning about preferences in qualitative settings is an important research area for knowledge representation and qualitative decision theory. The main objectives are to design expressive yet intuitive languages to model preferences, and to develop automated methods to reason about formal representations of preferences in these languages. The literature on the subject of preferences is vast. We refer the reader to articles in the special issue of the Artificial Intelligence Magazine (Goldsmith \& Junker, 2008) and to a recent monograph by Kaci (2011) for a thorough discussion of the area and for additional references.

Understanding when optimization problems are equivalent, in particular, when one can be interchanged with another within any larger context, is fundamental to any preference formalism. Speaking informally, optimization problems $P$ and $Q$ are interchangeable or strongly equivalent when for every optimization problem $R$ (context), $P \cup R$ and $Q \cup R$ define the same optimal models. Understanding when one optimization problem is equivalent to another in this sense is essential for preference analysis, modular preference representation, and rewriting techniques to simplify optimization problems into forms more amenable to processing, without changing any of their inherent properties. Let us consider a multi-agent setting, in which agents combine their preferences on some set of alternatives with the goal of identifying optimal ones. Can one agent in the ensemble be replaced with another so that the set of optimal alternatives is unaffected not only now, but also under any extension of the ensemble in the future? Strong equivalence of agents' optimization problems is precisely what is needed to guarantee this full interchangeability property!

The notion of strong equivalence is of general interest, by no means restricted to preference formalisms. In some cases, most notably for classical logic, it coincides with equivalence, the property of having the same models. However, if the semantics is not monotone, that is, extending the theory may introduce new models not only eliminate some, strong equivalence becomes a strictly stronger concept, and the one to adopt if theories being analyzed are to be placed within a larger context. The nonmonotonicity of the semantics is the salient feature of nonmonotonic logics (Marek \& Truszczyński, 1993) and strong equivalence of theories in nonmonotonic logics, especially logic programming with the answer-set semantics (Gelfond \& Lifschitz, 1991), was extensively studied in that setting (Lifschitz, Pearce, \& Valverde, 2001; Turner, 2003; Eiter, Fink, \& Woltran, 2007b). Preference formalisms also often behave nonmonotonically as adding a new preference may cause a non-optimal outcome (model) to become an optimal one. Thus, in preference formalisms, equivalence and strong equivalence are typically different notions. Accordingly, strong equivalence was studied for logic programs with rule preferences (Faber \& Konczak, 2006), programs with ordered disjunction (Faber, Tompits, \& Woltran, 2008) and programs with weak constraints (Eiter, Faber, Fink, \& Woltran, 2007a).

We extend the study of strong equivalence to the formalism of qualitative optimization problems. The formalism is motivated by the design of answer-set optimization (ASO) programs of Brewka et al. (2003). It borrows two key features from ASO programs that make it an attractive alternative to the preference modeling approaches based on logic programming that we mentioned above. First, following ASO programs, optimization problems provide a clear separation of hard constraints, which specify the space of feasible outcomes, and 
preferences (soft constraints) that impose a preference ordering on feasible outcomes. Second, optimization problems adopt the syntax and the semantics of preference rules of ASO programs that correspond closely to linguistic patterns of simple conditional preferences used by humans.

The separation of preference modules from hard constraints facilitates eliciting and representing preferences. It is also important for characterizing strong equivalence. When a clear separation is not present, like in logic programs with ordered disjunctions (Brewka, Niemelä, \& Syrjänen, 2004), strong equivalence characterizations are cumbersome as they have to account for complex and mostly implicit interactions between hard constraints and preferences. For optimization problems, which impose the separation, we have "onedimensional" forms of strong equivalence, in which only hard constraints or only preferences are added. These "one-dimensional" concepts are easier to study yet provide enough information to construct characterizations for the general case.

Main Contributions. Our main contribution can be summarized as follows.

- We propose a general framework of qualitative optimization problems, extending in several ways the formalism of ASO programs. We focus on two important instantiations of the framework, the classes of classical optimization (CO) problems and answer-set optimization (ASO) problems. The latter one directly generalizes ASO programs.

- We identify the problem of strong equivalence for theories in general preference formalisms. We point out that strong equivalence and equivalence do not coincide (in general) in preference formalisms and that it is the concept of strong equivalence that is fundamental for such issues as theory modularity, rewriting and simplification. Strong equivalence was studied earlier in the context of logic programs and logic programs extended with preferences on rules or on atoms in the heads of rules (and was similarly motivated). However, to the best of our knowledge, this is the first paper that studies strong equivalence for a more typical preference formalism that represents preferences in terms of "preferred properties" (modeled by formulas) and independently of constraints defining outcomes to compare. As such, it is of more relevance to the mainstream preference research than the previous studies.

- We characterize the concept of strong equivalence of optimization problems relative to changing selector modules. The characterization is independent of the semantics of generators and so, applies both to $\mathrm{CO}$ and ASP problems. We also characterize strong equivalence relative to changing generators (with preferences fixed). In this case, not surprisingly, the characterization depends on the semantics of generators. However, we show that the dependence is quite uniform, and involves a characterization of strong equivalence of generators relative to their underlying semantics, when they are considered on their own as propositional theories. Finally, we combine the characterizations of the "one-dimensional" concepts of strong equivalence into a characterization of the general "combined" notion.

- We develop our results for the case when preferences are ranked. In practice, preferences are commonly ranked due to the hierarchical structure of preference providers. 
The general case we study allows for additions of preferences of ranks from a specified interval $[i, j]$. This covers the case when only some segment in the hierarchy of preference providers is allowed to add preferences (top decision makers, middle management, low-level designers), as well as the case when there is no distinction between the importance of preferences (the non-ranked case).

- We establish the complexity of deciding whether two optimization problems are strongly equivalent relative to changing selectors, generators, or both. Our results show that these problems range from being co-NP- to $\Pi_{3}^{P}$-complete.

Organization. In the following section, we introduce the concept of an optimization problem and the necessary terminology, and define all equivalence problems we are interested in here. We also discuss the relationship of optimization problems to further formalisms from the literature, in particular to ASO programs. In Section 3 we provide our results for the case where selectors may vary but no new hard constraints are allowed. Section 4 in turn characterizes the strong equivalence notion, where preferences are unaffected but the generator parts are subject to change. In Section 5 we finally show how the characterizations obtained in the previous sections have to be combined in order to capture the general case of strong equivalence. Our complexity analysis is presented in Section 6, followed by a discussion of our results and considerations about future directions of research.

We present proof sketches and some of the simpler and not overly technical proofs in the main text to facilitate understanding of the results at the intuitive level. Detailed proofs can be found in the Appendix.

This article is a substantially extended version of an earlier published conference version (Faber, Truszczyński, \& Woltran, 2012).

\section{Optimization Problems}

In this section we provide the basic definitions of optimization problems in Section 2.1, followed in Section 2.2 by definitions of the strong equivalence notions on optimization problems that are studied in the remainder of the paper. Finally, in Section 2.3 we provide a discussion on related formalisms.

\subsection{Basic Definitions}

A qualitative optimization problem (an optimization problem, from now on) is an ordered pair $P=(T, S)$, where $T$ is called the generator and $S$ the selector. The role of the generator is to specify the family of outcomes to be compared. The role of the selector $S$ is to define a relation $\geq$ on the set of outcomes and, consequently, define the notion of an optimal outcome. The relation $\geq$ induces relations $>$ and $\approx$ : we define $I>J$ if $I \geq J$ and $J \nsupseteq I$, and $I \approx J$ if $I \geq J$ and $J \geq I$. For an optimization problem $P$, we write $P^{g}$ and $P^{s}$ to refer to its generator and selector, respectively.

Generators. As generators we use propositional theories in the language determined by a fixed countable universe (or alphabet) $\mathcal{U}$ of propositional variables that form atomic propositions, a Boolean constant $\perp$, and Boolean connectives $\wedge, \vee$ and $\rightarrow$, and where we define the constant $\top$, and the connectives $\neg$ and $\leftrightarrow$ in the usual way as $\top:=\neg \perp, \neg \phi:=$ 
$\phi \rightarrow \perp$, and $\phi \leftrightarrow \psi:=(\phi \rightarrow \psi) \wedge(\psi \rightarrow \phi)$, respectively. ${ }^{1}$ Models of the generator, as defined by the semantics used, represent outcomes of the corresponding optimization problem. We consider two quite different semantics for generators: the classical propositional logic semantics and the semantics of equilibrium models (Pearce, 1997). Thus, outcomes are either models or equilibrium models, depending on the semantics chosen. The first semantics is of interest due to the fundamental role and widespread use of classical propositional logic, in particular, as a means to describe constraints. Equilibrium models generalize answer sets of logic programs to the case of arbitrary propositional theories (Pearce, 1997; Ferraris, 2005) and are often referred to as answer sets. The semantics of equilibrium models is important due to the demonstrated effectiveness of logic programming with the semantics of answer sets for knowledge representation applications. We use the terms equilibrium models and answer sets interchangeably.

Throughout the paper, we represent interpretations as subsets of $\mathcal{U}$, which contain exactly those atomic propositions that are interpreted as true. We write $I \models \phi$ to state that an interpretation $I \subseteq \mathcal{U}$ is a (classical propositional) model of a formula $\phi$. Furthermore, we denote the set of classical models of a formula or theory $T$ by $\operatorname{Mod}(T)$.

Equilibrium models arise in the context of the propositional logic of here-and-there, or the logic HT for short (Heyting, 1930). We briefly recall here definitions of concepts, as well as properties of the logic HT that are directly relevant to our work. We refer to the papers by Pearce (1997) and Ferraris (2005) for further details.

The logic HT is a logic located between the intuitionistic and the classical logics. Interpretations in the logic HT are pairs $\langle I, J\rangle$ of standard propositional interpretations such that $I \subseteq J$. We write $\langle I, J\rangle \models_{H T} \phi$ to denote that a formula $\phi$ holds in an interpretation $\langle I, J\rangle$ in the logic HT. The relation $\models_{H T}$ is defined recursively as follows:

1. $\langle I, J\rangle \not \nvdash_{H T} \perp$

2. for an atom $a,\langle I, J\rangle=_{H T} a$ precisely when $a \in I$

3. $\langle I, J\rangle \models_{H T} \phi \vee \psi$ if $\langle I, J\rangle \models_{H T} \phi$ or $\langle I, J\rangle \models_{H T} \psi$

4. $\langle I, J\rangle \models_{H T} \phi \wedge \psi$ if $\langle I, J\rangle \models_{H T} \phi$ and $\langle I, J\rangle \models_{H T} \psi$

5. $\langle I, J\rangle \mid=H T \phi \rightarrow \psi$ if $J \mid=\phi \rightarrow \psi$ (classical satisfiability), and $\langle I, J\rangle \mid \models_{H T} \phi$ or $\langle I, J\rangle \models_{H T} \psi$.

An equilibrium model or answer set of a propositional theory $T$ is a standard interpretation $I$ such that $\langle I, I\rangle \models_{H T} T$ and for every proper subset $J$ of $I,\left\langle J,\left.I\right|_{H T} T\right.$. Answer sets of a propositional theory $T$ are also classical models of $T$. The converse is not true in general. We denote the set of all answer sets of a theory $T$ by $A S(T)$, and the set of all HT-models of $T$ by $\operatorname{Mod}_{H T}(T)$, that is, $\operatorname{Mod}_{H T}(T)=\left\{\langle I, J\rangle \mid I \subseteq J,\langle I, J\rangle \models_{H T} T\right\}$.

For each of the semantics there are two natural concepts of equivalence. Two theories $T_{1}$ and $T_{2}$ are equivalent if they have the same models (classical or equilibrium, respectively). They are strongly equivalent if for every theory $S, T_{1} \cup S$ and $T_{2} \cup S$ have the same models (again, classical or equilibrium, respectively).

1. While the choice of primitive connectives is not common for the language of classical propositional logic, it is standard for the of logic here-and-there which underlies the answer-set semantics. 
For classical semantics, strong equivalence and equivalence coincide. It is not so for the semantics of equilibrium models. The result by Lifschitz et al. (2001) states that two theories $T_{1}$ and $T_{2}$ are strongly equivalent for equilibrium models if and only if $T_{1}$ and $T_{2}$ are equivalent in the logic HT, that is, $\operatorname{Mod}_{H T}\left(T_{1}\right)=\operatorname{Mod}_{H T}\left(T_{2}\right)$. We will now illustrate the notions of HT-models and equilibrium models, and relate the latter to the classical ones. Here and in all other examples, we consider only classical and HT-models over the alphabet consisting of atoms explicitly mentioned in the theories discussed. That is sufficient to determine all equilibrium models on the one hand (which happen to consist only of atoms that are mentioned) and, on the other, to show that they differ from classical ones.

Example 1 Let us consider the theory $T_{a}=\{a \rightarrow a\}$. The classical models of $T_{a}$ (under the restriction mentioned above) are $\emptyset$ and $\{a\}$, so a being true and a being false are both possible outcomes. The HT-models (again, under the same restriction) are $\langle\emptyset, \emptyset\rangle,\langle\emptyset,\{a\}\rangle$, and $\langle\{a\},\{a\}\rangle$. Hence, there is only one answer set (equilibrium model) $\emptyset$. The other possible candidate, $\{a\}$, is not an answer set. While $\langle\{a\},\{a\}\rangle \models_{H T} T_{a}$ holds, also $\langle\emptyset,\{a\}\rangle \models_{H T} T_{a}$ does. Thus, intuitively, the theory does not contain any "cause" for a to hold.

Next, let us consider the theory $T_{b}=\{a \vee b\}$. The classical models are $\{a\},\{b\}$ and $\{a, b\}$, and the HT-models are $\langle\{a\},\{a\}\rangle,\langle\{b\},\{b\}\rangle,\langle\{a\},\{a, b\}\rangle,\langle\{b\},\{a, b\}\rangle$ and $\langle\{a, b\},\{a, b\}\rangle$. The answer sets are therefore $\{a\}$ and $\{b\}$, but not $\{a, b\}$. Again the intuition is that the theory does not contain a "cause" for $a$ and $b$ to hold simultaneously.

Finally, let us consider the theory $T_{c}=\{(\neg a \rightarrow b) \wedge(\neg b \rightarrow a)\}$. The classical models are the same as for $T_{b}$, that is, $\operatorname{Mod}\left(T_{c}\right)=\{\{a\},\{b\},\{a, b\}\}$. We also have $\operatorname{Mod}_{H T}\left(T_{c}\right)=$ $\{\langle\{a\},\{a\}\rangle,\langle\{b\},\{b\}\rangle,\langle\{a\},\{a, b\}\rangle,\langle\{b\},\{a, b\}\rangle,\langle\{a, b\},\{a, b\}\rangle,\langle\emptyset,\{a, b\}\rangle\}=\operatorname{Mod}_{H T}\left(T_{b}\right) \cup$ $\{\langle\emptyset,\{a, b\}\rangle\}$. The answer sets are again the same as for $T_{b}: A S\left(T_{c}\right)=\{\{a\},\{b\}\}$.

We observe that $T_{b}$ and $T_{c}$ are equivalent for both the classical and equilibrium setting (they have the same classical and equilibrium models). The former implies that they are also strongly equivalent in the classical setting. However, they are not strongly equivalent in the equilibrium setting because $\operatorname{Mod}_{H T}\left(T_{b}\right) \neq \operatorname{Mod}_{H T}\left(T_{c}\right)$ (cf. the characterization of strong equivalence under the equilibrium semantics by Lifschitz et al. (2001)). And indeed, for $S=\{a \rightarrow b, b \rightarrow a\}$, we obtain $A S\left(T_{b} \cup S\right)=\{\{a, b\}\}$, while $A S\left(T_{c} \cup S\right)=\emptyset$.

We recall that optimization problems under the classical interpretation of generators are referred to as classical optimization problems or CO problems, and when we use the answer-set semantics for generators, we speak about answer-set optimization problems or ASO problems.

Selectors. We follow the definitions of preference modules in ASO programs (Brewka et al., 2003), adjusting the terminology to our more general setting. A selector is a finite set of ranked preference rules

$$
\phi_{1}>\cdots>\phi_{k} \stackrel{j}{\leftarrow} \psi
$$

where $k$ and $j$ are positive integers, and $\phi_{i}, 1 \leq i \leq k$, and $\psi$ are propositional formulas over $\mathcal{U}$. For a rule $r$ of the form (1), the number $j$ is the rank of $r$, denoted by $\operatorname{rank}(r)$, $h d(r)=\left\{\phi_{1}, \ldots, \phi_{k}\right\}$ is the head of $r$ and $\psi$ is the body (the condition) of $r, b d(r)$. Moreover, we write $h d_{i}(r)$ to refer to formula $\phi_{i}$.

If $\operatorname{rank}(r)=1$ for every preference rule $r$ in a selector $S$, then $S$ is a simple selector. Otherwise, $S$ is ranked. We often omit "1" from the notation " $\leftarrow$ " for simple selectors. For 
a selector $S$, and $i, j \in\{0,1,2, \ldots\} \cup\{\infty\}$, we define $S_{[i, j]}=\{r \in S \mid i \leq \operatorname{rank}(r) \leq j\}$ (where we assume that for every integer $k, k<\infty$ ) and write $[i, j]$ for the rank interval $\{k \mid k$ an integer, $i \leq k \leq j\}$. We extend this notation to optimization problems. For $P=(T, S)$ and a rank interval $[i, j]$, we set $P_{[i, j]}=\left(T, S_{[i, j]}\right)$. For some rank intervals we use shorthands, for example $=i$ for $[i, i],<i$ for $[1, i-1], \geq i$ for $[i, \infty]$, and similar.

For an interpretation $I$, a satisfaction degree of a preference rule $r$ is $v_{I}(r)=\min \{i|I|=$ $\left.h d_{i}(r)\right\}$, if $I \models b d(r)$ and $I \models \bigvee h d(r)$; otherwise, the rule is irrelevant to $I$, and $v_{I}(r)=1$. Intuitively, the lower the satisfaction degree the better the outcome. Thus, a preference rule $\phi_{1}>\cdots>\phi_{k} \leftarrow \psi$ can informally be read as follows: "irrelevant outcomes (those not satisfying $\psi$, or not satisfying any $\phi_{i}$ ) and outcomes satisfying $\phi_{1}$ are most preferred, followed by outcomes satisfying $\phi_{2}$, and then outcomes satisfying $\phi_{3}$, etc." We note that Brewka et al. (2003) represented the satisfaction degree of an irrelevant rule by a special non-numeric degree, treated as being equivalent to 1 . The difference is immaterial and the two approaches are equivalent.

Selectors determine a preference relation on interpretations. Given interpretations $I$ and $J$ and a simple selector $S, I \geq S J$ holds precisely when for all $r \in S, v_{I}(r) \leq v_{J}(r)$. Therefore, $I>^{S} J$ holds if and only if $I \geq^{S} J$ and there exists $r \in S$ such that $v_{I}(r)<v_{J}(r)$; $I \approx^{S} J$ holds if and only if for every $r \in S, v_{I}(r)=v_{J}(r)$.

Given a ranked selector $S$, we define $I \geq^{S} J$ if for every preference rule $r \in S, v_{I}(r)=$ $v_{J}(r)$, or if there is a rule $r^{\prime} \in S$ such that the following three conditions hold:

1. $v_{I}\left(r^{\prime}\right)<v_{J}\left(r^{\prime}\right)$

2. for every $r \in S$ of the same rank as $r^{\prime}, v_{I}(r) \leq v_{J}(r)$

3. for every $r \in S$ of smaller rank than $r^{\prime}, v_{I}(r)=v_{J}(r)$.

Moreover, $I>^{S} J$ if and only if there is a rule $r^{\prime}$ for which the three conditions above hold, and $I \approx^{S} J$ if and only if for every $r \in S, v_{I}(r)=v_{J}(r)$. Given an optimization problem $P=(T, S)$, we often write $\geq^{P}$ for $\geq^{S}$ (and similarly for $>$ and $\approx$ ). Furthermore, for a set $V \subseteq 2^{\mathcal{U}}$ and a relation $\succ$ (like $>, \geq$, or $\approx$ ) over $2^{\mathcal{U}}$, we write $\succ_{V}$ for the restriction of $\succ$ to $V$, that is, $\succ_{V}=\{(A, B) \in \succ \mid A, B \in V\}$. The relationship between equalities of $\geq,>$, and $\approx$ of two optimization problems is as follows.

Lemma 1 For all optimization problems $P$ and $Q$, and every set $V \subseteq 2^{\mathcal{U}}, \geq_{V}^{P}=\geq_{V}^{Q}$ implies $>_{V}^{P}=>_{V}^{Q}$ and $\approx_{V}^{P}=\approx_{V}^{Q}$.

Proof. Suppose $\geq_{V}^{P}=\geq_{V}^{Q}$ and let $I, J$ be interpretations such that $I>_{V}^{P} J$. By definition, $I \geq_{V}^{P} J$ but $J \nsupseteq_{V}^{P} I$. By assumption $I \geq_{V}^{Q} J$ and $J \geq_{V}^{Q} I$, implying $I>_{V}^{Q} J$. In case $I \approx_{V}^{P} J$ we have $I \geq_{V}^{P} J$ and $J \geq_{V}^{P} I$. By assumption $I \geq_{V}^{Q} J$ and $J \geq_{V}^{Q} I$ hold as well and we conclude $I \approx_{V}^{Q} J$. The other direction $\left(I>>_{V}^{Q} J\right.$ implies $I>_{V}^{P} J$, and $I \approx_{V}^{Q} J$ implies $\left.I \approx_{V}^{P} J\right)$ is analogous.

Some aspects of ASO selectors require additional discussion. First, a preference rule may be irrelevant to an outcome. This is the case when the outcome does not satisfy the condition of a rule or, when it does, if it does not satisfy any formula in the head of the rule. In such cases, we define the outcome to be most desirable with respect to the rule. In making this choice, we followed the original definition (Brewka et al., 2003) (modulo a 
minor simplification mentioned earlier). Obviously, other choices could be considered, too. For instance, we could define irrelevant outcomes to be the least desirable with respect to the rule. We could also restrict attention only to selectors that do no permit irrelevance at all (a preference rule does not allow irrelevance if it has no body and if the disjunction of all options in the head is a tautology). This would eliminate the need to address that issue altogether, however, at the price of a more constraining definintion of selector rules.

Ultimately, the question what is the right design choice here is of secondary importance as the semantics of preference rules we adopted provides us the flexibility to represent other possible definitions. In particular, we note that the semantics of the rule

$$
\phi_{1}>\ldots>\phi_{n} \leftarrow \psi
$$

is the same as that of the rule

$$
\phi_{1} \wedge \psi>\ldots>\phi_{n} \wedge \psi \leftarrow \text {. }
$$

In other words, the conditions (the rule bodies) are only a modeling device making preference rules better correspond to conditional preferences expressed in natural language. They can be "compiled away." As for the second type of irrelevance, the formalism of selectors allows the user to override the default we adopted. We can make our adopted design choice explicit, that is, making outcomes not satisfying any of the options in the head explicitly the most desirable. Intuitively, it is sufficient to rewrite the rule (without body, since bodies can be removed, as shown earlier)

$$
\phi_{1}>\ldots>\phi_{n} \leftarrow
$$

to

$$
\phi_{1} \vee \neg\left(\phi_{1} \vee \ldots \vee \phi_{n}\right)>\ldots>\phi_{n} \leftarrow
$$

or, equivalently,

$$
\phi_{1} \vee \neg\left(\phi_{2} \vee \ldots \vee \phi_{n}\right)>\ldots>\phi_{n} \leftarrow
$$

while the following rewriting makes them least desirable:

$$
\phi_{1}>\ldots>\phi_{n} \vee \neg\left(\phi_{1} \vee \ldots \vee \phi_{n}\right) \leftarrow
$$

or, equivalently,

$$
\phi_{1}>\ldots>\phi_{n} \vee \neg\left(\phi_{1} \vee \ldots \vee \phi_{n-1}\right) \leftarrow .
$$

Another question concerns rules with only one option in the head. Intuitively, given our semantics they should not be important, because their satisfaction degree is always 1 . Indeed, Corollary 12 later in the paper provides a formal result that confirms this statement.

Optimal (preferred) outcomes. For an optimization problem $P, \mu(P)$ denotes the set of all outcomes of $P$, that is, the set of all models (under the selected semantics) of the generator of $P$. Thus, $\mu(P)$ stands for all models of $P$ in the framework of CO problems and for all answer sets of $P$, when ASO problems are considered. A model $I \in \mu(P)$ is optimal or preferred for $P$ if there is no model $J \in \mu(P)$ such that $J>^{P} I$. We denote the set of all preferred models of $P$ by $\pi(P)$.

The following lemma asserts that if the preference relation of two optimization problems is equal on their sets of outcomes, then the preferred models coincide. This result follows 
immediately from the definitions and will be useful in the sequel. Its statement brings up a subtle notational issue. Formally, a (strict) preorder is a pair $(D,>)$, where $D$ is a set (the domain of the preorder) and $>$ is a transitive acyclic binary relation on $D$ (the preorder relation). Two preorders are equal if they have the same domain and the same relation on that domain. Typically, whenever the domain is understood, we refer to preorders just by pointing to their relation symbols. Often, however, we write $>_{D}$ for the preorder relation symbol to make the domain explicit in the notation. This is what we do in the statement of the result below.

Lemma 2 Let $P$ and $Q$ be optimization problems with $>_{\mu(P)}^{P}=>_{\mu(Q)}^{Q}$. Then, $\pi(P)=\pi(Q)$.

Proof. As observed above, the equality of preorders implies the equality of the domains. In our case, the equality $>_{\mu(P)}^{P}=>_{\mu(Q)}^{Q}$ implies $\mu(P)=\mu(Q)$. Hence, for each $I, J \in \mu(P)=\mu(Q), I>^{P} J$ iff $I>^{Q} J$. The result follows now directly from the definition of preferred outcomes.

We also observe that eliminating rules of large ranks can only make unpreferred outcomes preferred, and never make preferred outcomes unpreferred. We recall that, for a given optimization problem $P=(T, S), P_{<i}=P_{[1, i-1]}=\left(T, S_{[1, i-1]}\right)$ is the corresponding optimization problem with all rules with rank $i$ or higher removed.

Lemma 3 For every optimization problem $P$ and every $i \geq 1, \pi\left(P_{<i}\right) \supseteq \pi(P)$.

Proof. Let us assume that $I \notin \pi\left(P_{<i}\right)$. In case $I \notin \mu\left(P_{<i}\right)$, we have $I \notin \mu(P)$, and $I \notin \pi(P)$

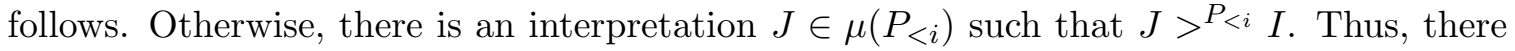
is a rule $r \in P_{<i}^{s}$, say of rank $j$, such that (i) $v_{J}(r)<v_{I}(r)$; (ii) for every $r^{\prime} \in P_{<i}^{s}$ with the rank $j, v_{J}\left(r^{\prime}\right) \leq v_{I}\left(r^{\prime}\right)$; and (iii) for every $r^{\prime} \in P_{<i}^{s}$ with rank less then $j, v_{J}\left(r^{\prime}\right)=v_{I}\left(r^{\prime}\right)$. We note that, since $\mu\left(P_{<i}\right)=\mu(P), J \in \mu(P)$. Moreover, $j<i$ and so the sets of rules with ranks less than or equal to $j$ in $P^{s}$ and $P_{<i}^{s}$ coincide. Thus, $J>^{P} I$ follows and, consequently, $I \notin \pi(P)$.

\subsection{Notions of Equivalence}

We define the union of optimization problems as expected, that is, for $P_{1}=\left(T_{1}, S_{1}\right)$ and $P_{2}=\left(T_{2}, S_{2}\right)$, we set $P_{1} \cup P_{2}=\left(T_{1} \cup T_{2}, S_{1} \cup S_{2}\right)$. Two optimization problems $P_{1}$ and $P_{2}$ are strongly equivalent with respect to a class $\mathcal{R}$ of optimization problems (referred to as a class of contexts or simply contexts) if for every optimization problem $R \in \mathcal{R}$, $\pi\left(P_{1} \cup R\right)=\pi\left(P_{2} \cup R\right)$.

We consider three general classes of contexts. First and foremost, we are interested in the class $\mathcal{L}_{\mathcal{U}}$ of all optimization problems over $\mathcal{U}$. We also consider the families $\mathcal{L}_{\mathcal{U}}^{g}$ and $\mathcal{L}_{\mathcal{U}}^{s}$ of all optimization problems of the form $(T, \emptyset)$ and $(\emptyset, S)$, respectively. The first class consists of optimization problems which, when added to any other problem, can affect the set of feasible outcomes but cannot affect the preference relation. We call such optimization problems generator problems. The second class consists of optimization problems which, when added to any other problem, do not change the set of feasible outcomes but change 
(in general) the preference relation. We call such optimization problems selector problems. These "one-dimensional" contexts provide essential insights into the general case. For these two classes, we speak of strong gen-equivalence, denoted $\equiv_{g}$, and strong sel-equivalence, denoted $\equiv^{s}$, respectively. For the general class $\mathcal{L}_{\mathcal{U}}$ of all optimization problems we simply speak of strong equivalence, denoted $\equiv_{g}^{s}$.

We recall that the notion of strong equivalence is, by definition, underlying the replacement property. In fact, for an optimization problem $P=(T, S)$ containing a subproblem $Q=\left(T^{\prime}, S^{\prime}\right)$ (i.e. $T^{\prime} \subseteq T$ and $S^{\prime} \subseteq S$ ) we can guarantee that $Q$ can be replaced in $P$ by another subproblem $R$ without changing the optimal outcomes, if $Q \equiv_{g}^{s} R$. Indeed, if $Q \equiv_{g}^{s} R$ holds, one can faithfully replace $Q$ by $R$ in any optimization problem (otherwise we would have $\pi\left(Q \cup P^{\prime}\right) \neq \pi\left(R \cup P^{\prime}\right)$ for some $\left.P^{\prime} \in \mathcal{L}_{\mathcal{U}}\right)$.

Constraining ranks of rules in selectors gives rise to additional classes of contexts parameterized by rank intervals $[i, j]$ :

1. $\mathcal{L}_{\mathcal{U}}^{s,[i, j]}=\left\{(\emptyset, S) \in \mathcal{L}_{\mathcal{U}}^{s} \mid S=S_{[i, j]}\right\}$

2. $\mathcal{L}_{\mathcal{U}}^{[i, j]}=\left\{(T, S) \in \mathcal{L}_{\mathcal{U}} \mid S=S_{[i, j]}\right\}$

The first class of contexts gives rise to strong sel-equivalence with respect to rules of rank in $[i, j]$, denoted by $\equiv^{s,[i, j]}$. The second class of contexts yields the concept of strong equivalence with respect to rules of rank in $[i, j]$. We denote it by $\equiv_{g}^{s,[i, j]}$. We call problems in the class $\mathcal{L}_{\mathcal{U}}^{=1}=\mathcal{L}_{\mathcal{U}}^{[1,1]}$ simple optimization problems.

\subsection{Relation to Other Preference Formalisms}

Optimization problems are most closely related to ASO programs (Brewka et al., 2003). The formalism of optimization problems extends ASO programs in several ways. First, the generators of optimization problems are arbitrary propositional theories. Under the semantics of equilibrium models, the generators properly extend logic programs with the answer-set semantics, which are used as generators in ASO programs. Second, the selectors of optimization problems use arbitrary propositional formulas in the heads of preference rules, as well as for conditions in their bodies, which again generalizes the selectors of ASO programs. Finally, optimization problems explicitly allow for alternative semantics of generators, a possibility mentioned but not pursued by Brewka et al. (2003).

As we already noted in the introduction there is vast literature on preference representation and reasoning (The special issue of Artificial Intelligence Magazine, Goldsmith \& Junker, 2008, and the monograph by Kaci, 2011, are two comprehensive sources of relevant references. For a survey on preference approaches on top of nonmonotonic formalisms, see Delgrande, Schaub, Tompits, \& Wang, 2004). Discussing it goes beyond the scope of the present paper, especially as the problem that we focus on here (strong equivalence) has not been considered much in the preference research before, and there are essentially no relevant earlier results except those we already mentioned in the introduction (Faber \& Konczak, 2006; Faber et al., 2008; Eiter et al., 2007a). Nevertheless, since our work uses the preference formalism of ASO problems, an extension of the formalism of ASO programs by Brewka et al. (2003) that has not received as much attention in the preference research as some others, we will make a few comments on our choice. 
First, ASO problems are explicit about the constraints that must not be violated (the generator part) and preferences, that is, weaker constraints that only make some outcomes more desirable than others (the selector part). In that ASO problems match well with practical settings, as typically both kinds of constraints are at play. For instance, in a product configuration problem there are physical constraints limiting the space of available possibilities (not every type of engine can be put in a small family sedan, moon roof is not available with the basic engine option, etc.), as well as user preferences that describe what the user would like to have if possible. Preferential reasoning (optimization) in the presence of (hard) constraints has received substantial attention. A representative approach in which CP-nets (Boutilier, Brafman, Domshlak, Hoos, \& Poole, 2003) are combined with constraints was described in the paper by Boutilier, Brafman, Domshlak, Hoos, and Poole (2004).

The choice of propositional logic to represent constraints (the generator part in our formalism) is standard. However, in contrast with other approaches, in addition to the classical semantics we also consider an appealing alternative, the semantics of answer sets. This is important as the resulting formalism of answer-set programming (Marek \& Truszczyński, 1999; Niemelä, 1999) is steadily gaining on acceptance as a constraint language and is supported by ever improving computational tools (Calimeri, Ianni, Krennwallner, \& Ricca, 2012).

On the other hand, our choice of the formalism for the selector part is less obvious. There are several reasons that motivated us. The first, and the one we already mentioned earlier, is that preference rules have a natural reading agreeing well with the linguistic patterns that humans use when formulating qualitative conditional preferences. Second, as demonstrated in the original work where ASO selectors were introduced (Brewka et al., 2003), they can be used to approximate preference relations that are defined by CP-nets (Boutilier et al., 2003), one of the most broadly studied qualitative preference systems, but has better computational properties. For instance, the dominance problem is in $\mathrm{P}$ as opposed to being NP-hard or even PSPACE-complete for some generalized classes of CP-nets (Goldsmith, Lang, Truszczyński, \& Wilson, 2008).

Third, individual preference rules are closely related to one of the standard approaches to representing preferences based on possibilistic logic. In that approach (we only give the most basic details here, for a more comprehensive discussion we refer to Kaci, 2011, Ch. 3.3.3), a preference theory consists of formulas, each with a distinct rank (the assumption that ranks are distinct is not limiting as formulas with repeating ranks can be "conjuncted" into a single formula of that rank). The quality of an outcome is given by its score defined as the minimum rank of a formula that the outcome does not satisfy $(\infty$, if all formulas are satisfied). The higher the score, the better the outcome. Let $\left\{\phi_{1}, \ldots, \phi_{n}\right\}$ be a preference theory, with the index $i$ in $\phi_{i}$ representing the rank of $\phi_{i}$. It is clear that the preference semantics of that theory as described above is precisely captured by our preference rule

$$
\psi_{n}>\psi_{n-1}>\ldots>\psi_{1}>\neg \psi_{1} \leftarrow
$$

where $\psi_{i}=\phi_{1} \wedge \ldots \wedge \phi_{i}$, for $i=1, \ldots, n$. Thus, ASO problems subsume the preference formalism based on possibilistic logic.

Finally, the selector part of ASO problems typically consists of several preference rules and these rules may have different ranks. That allows us to model preferences coming from different sources and having different importance. In such cases, the main issue is that 
of integrating these individual preferences into a single order. There is no single broadly accepted way to do so. The approach used by our formalism boils down to the Pareto principle, arguably the common core of all such integration principles. Accordingly, our formalism allows conflicting rules in the selector (for instance, $a>b \leftarrow$ and $b>a \leftarrow$ ) but leaves such conflicts unresolved resulting in incomparability. As for the ranks, the lower the rank, the more important the rule is. Rules of less importance are used to compare outcomes only if the rules of more importance do not distinguish them. This way of handling ranks is natural and shows up in many preference formalisms.

One prominent example in this context is prioritized (propositional) circumscription (Lifschitz, 1985), where minimization (of certain atoms in models of a formula) is defined with respect to classes of atoms of different priority. Formally, let $T$ be a theory over atoms $A$ and $\left(P_{1}, \ldots, P_{n}, V, F\right)$ be a partition on $A$. Then, a model $M \in \operatorname{Mod}(T)$ is called $\left(P_{1}, \ldots, P_{n}, V, F\right)$-minimal if there is no $N \in \operatorname{Mod}(T)$, such that (i) $N \cap\left(P_{1} \cup \cdots \cup P_{i-1}\right)=$ $M \cap\left(P_{1} \cup \cdots \cup P_{i-1}\right)$ and $N \cap P_{i} \subset M \cap P_{i}$ for some $1 \leq i \leq n$, and (ii) $N \cap F=M \cap F$. The intuition behind the definition is that atoms in $P=\bigcup_{i} P_{i}$ are to be minimized, with the assignments to $V$ allowed to vary, while assignments to $F$ are to be kept fixed. Atoms in $P$ are minimized such that those in $P_{1}$ have the highest priority followed by those in $P_{2}$, etc. The relation to ranks is quite obvious. One can show that the $\left(P_{1}, \ldots, P_{n}, V, F\right)$-minimal models of a theory $T$ coincide with the preferred outcomes of the CO problem $X=(T, S)$, where the selector $S$ is given by

$$
\begin{aligned}
S= & \{f>\neg f \stackrel{1}{\leftarrow} \top \mid f \in F\} \cup\{\neg f>f \stackrel{1}{\leftarrow} \top \mid f \in F\} \\
& \left.\cup\left\{\neg p>p \stackrel{i}{\leftarrow} \top \mid p \in P_{i}, 1 \leq i \leq n\right\}\right) .
\end{aligned}
$$

Indeed preference rules in the first two sets ensure that only interpretations with the same fixed part are comparable, and preference rules in the last group precisely reflect the prioritized process of minimization of atoms in $P_{1} \cup \ldots \cup P_{n}$.

Let us just finally mention here that our formalism of optimization problems gives not only a handle for classical prioritized circumscription, but also for circumscription put on top of logic programs (this is not meaningless, as in the forms currently prevalent in answer set programming, answer sets are not necessarily minimal models, see for instance Simons, Niemelä, \& Soininen, 2002). To this end it suffices to apply the embedding using ASO problems instead of CO problems.

\section{Strong Sel-Equivalence}

We start by analyzing the case of strong sel-equivalence that turns out to be the core case for our study. Indeed, characterizations of strong sel-equivalence naturally imply characterizations for the general case thanks to the following simple observation.

Proposition 4 Let $P$ and $Q$ be optimization problems (either under classical or answer-set semantics for the generators) and $[i, j]$ a rank interval. Then $P \equiv_{g}^{s,[i, j]} Q$ if and only if for every generator $R \in \mathcal{L}_{\mathcal{U}}^{g}, P \cup R \equiv{ }^{s,[i, j]} Q \cup R$.

Proof. $(\Rightarrow)$ Let $R \in \mathcal{L}_{\mathcal{U}}^{g}$. Since $P \equiv_{g}^{s,[i, j]} Q, P \cup R \equiv_{g}^{s,[i, j]} Q \cup R$ and so, $P \cup R \equiv{ }^{s,[i, j]} Q \cup R$. 
$(\Leftarrow)$ Let $R$ be any optimization problem in $\mathcal{L}_{\mathcal{U}}^{[i, j]}$. We have $P \cup R=\left(P \cup\left(R^{g}, \emptyset\right)\right) \cup\left(\emptyset, R^{s}\right)$ and $Q \cup R=\left(Q \cup\left(R^{g}, \emptyset\right)\right) \cup\left(\emptyset, R^{s}\right)$. Moreover, by the assumption we have that $P \cup\left(R^{g}, \emptyset\right) \equiv^{s,[i, j]}$ $Q \cup\left(R^{g}, \emptyset\right)$. Thus,

$$
\pi\left(\left(P \cup\left(R^{g}, \emptyset\right)\right) \cup\left(\emptyset, R^{s}\right)\right)=\pi\left(\left(Q \cup\left(R^{g}, \emptyset\right)\right) \cup\left(\emptyset, R^{s}\right)\right) .
$$

It follows that $\pi(P \cup R)=\pi(Q \cup R)$ and, consequently, that $P \equiv_{g}^{s,[i, j]} Q$.

Furthermore, the set of outcomes of an optimization problem $P$ is unaffected by changes in the selector module. It follows that the choice of the semantics for generators does not matter for characterizations of strong sel-equivalence. Thus, whenever in this section we refer to the set of outcomes of an optimization problem $P$, we use the notation $\mu(P)$, and not the more specific one, $\operatorname{Mod}\left(P^{g}\right)$ or $A S\left(P^{g}\right)$, that applies to $\mathrm{CO}$ and ASO problems, respectively.

To formally state the subsequent results, we need one more auxiliary notation. For an optimization problem $P$, we define $\operatorname{diff}^{P}(I, J)$ to be the largest $k$ such that $I \approx^{P_{<k}} J$. If for every $k$ we have $I \approx^{P}<k$, then we set $\operatorname{diff}^{P}(I, J)=\infty$. It is clear that $\operatorname{diff}^{P}(I, J)$ is well-defined. Moreover, as $I \approx^{P<1} J$, $\operatorname{diff}^{P}(I, J) \geq 1$. The following lemma characterizes the relation $>P \cup Q$ for ranked optimization problems $P$ and $Q$.

Lemma 5 Let $P$ and $Q$ be optimization problems, and $I, J$ be interpretations. Then, $I>P \cup Q J$ holds if and only if one of the following conditions holds:

1. $\operatorname{diff}^{P}(I, J)<\operatorname{diff}^{Q}(I, J)$ and $I>^{P} J$;

2. $\operatorname{diff}^{P}(I, J)>\operatorname{diff}^{Q}(I, J)$ and $I>^{Q} J$;

3. $\operatorname{diff}^{P}(I, J)=\operatorname{diff}^{Q}(I, J), I>^{P} J$ and $I>^{Q} J$.

Proof. The "if" direction is evident. To prove the "only-if" direction, we note that the cases $\operatorname{diff}^{P}(I, J)<\operatorname{diff}^{Q}(I, J)$ and $\operatorname{diff}^{P}(I, J)>\operatorname{diff}^{Q}(I, J)$ are obvious, too. Thus, let us assume $\operatorname{diff}^{P}(I, J)=\operatorname{diff}^{Q}(I, J)=i$. Clearly, $i<\infty$ (otherwise, $I \approx P \cup Q \quad J$, contrary to the assumption). It follows that for every rule $r \in P^{s} \cup Q^{s}$ of rank less than $i, v_{I}(r)=v_{J}(r)$. Next, for every $r \in P^{s} \cup Q^{s}$ of rank $i, v_{I}(r) \leq v_{J}(r)$. Finally, there are rules $r \in P^{s}$ and $r^{\prime} \in Q^{s}$, each of rank $i$ such that $v_{I}(r) \neq v_{J}(r)$ and $v_{I}\left(r^{\prime}\right) \neq v_{J}\left(r^{\prime}\right)$ (since $\operatorname{diff}^{P}(I, J)=i$ and $\left.\operatorname{diff}^{Q}(I, J)=i\right)$. It follows that $v_{I}(r)<v_{J}(r)$ and $v_{I}\left(r^{\prime}\right)<v_{J}\left(r^{\prime}\right)$. Thus, $I>^{P} J$ and $I>^{Q} J$, as needed.

Our first main result concerns strong sel-equivalence relative to selectors consisting of preference rules of ranks in a rank interval $[i, j]$. Special cases for strong sel-equivalence will follow as corollaries.

Considering strong sel-equivalence means that preference rules may be added to optimization problems. There are three main effects of doing so: outcomes that are equally good may become strictly comparable, strict comparability may be turned into incomparability, and the order of strict comparability may be reversed. To illustrate these phenomena, we show how they may affect strong sel-equivalence using the forthcoming examples. Importantly, they lead us towards conditions that are necessary for strong sel-equivalence and that motivate our characterization of that property that we formally state in Theorem 6 . 
Example 2 Let $P_{1}=\left(T_{1}, S_{1}\right)$, where $T_{1}$ is a theory "generating" exactly two outcomes $\{a\}$ and $\{b\}$ (for example $\{a \leftrightarrow \neg b\}$ ) and $S_{1}=\emptyset$ is empty. Clearly, $\pi\left(P_{1}\right)=\{\{a\},\{b\}\}$, because $\{a\}$ and $\{b\}$ are equally good with respect to $\geq^{P_{1}}$. It is possible to make them comparable by adding new preference rules. For example, let $R_{1}=(\emptyset,\{a>b \stackrel{i}{\leftarrow}\})$, where $i \geq 1$. Now, $\{a\}>^{P_{1} \cup R_{1}}\{b\}$ and thus $\pi\left(P_{1} \cup R_{1}\right)=\{\{a\}\}$. It is evident that for any pair of equally good interpretations $I, J$ one can find a context consisting only of preference rules that will make $I$ strictly preferred to $J$ (each new rule should have $I$ at least as preferred as $J$ and one of them should strictly prefer I to J), and that the precise ranks in such a context are not of importance.

Example 3 Let $P_{2}=\left(T_{1}, S_{2}\right)$, where $T_{1}$ is a theory generating exactly two outcomes $\{a\}$ and $\{b\}$, and $S_{2}=\{a>b \stackrel{i}{\leftarrow}\}$ for some rank $i \geq 1$. Clearly, $\{a\}>^{P_{2}}\{b\}$ and therefore $\pi\left(P_{2}\right)=\{\{a\}\}$. It is possible to make $\{a\}$ and $\{b\}$ incomparable by adding an appropriate context, for example $R_{2}=(\emptyset,\{b>a \stackrel{i}{\leftarrow}\})$. We obtain $\{a\} \ngtr^{P_{2} \cup R_{2}}\{b\}$ and $\{b\} \ngtr^{P_{2} \cup R_{2}}\{a\}$, thus $\pi\left(P_{2} \cup R_{2}\right)=\{\{a\},\{b\}\}$.

It is important to note that the rank of the context preference rule must be exactly equal to the rank of the original preference rule in order to achieve this effect, otherwise one preference rule would override the other. In general, for any pair of strictly comparable interpretations $I, J$ one can find an appropriate context that makes $I$ and $J$ incomparable, but in contrast to Example 2, the context must make use of rules of particular ranks.

Example 4 Let $P_{3}=\left(T_{1}, S_{3}\right)$, where $T_{1}$ is a theory admitting exactly two outcomes $\{a\}$ and $\{b\}$, and $S_{3}=\{a>b \stackrel{i}{\leftarrow}\}$ for some rank $i \geq 2$. Clearly, $\{a\}>^{P_{3}}\{b\}$ and therefore $\pi\left(P_{3}\right)=\{\{a\}\}$. It is possible to reverse the comparability of $\{a\}$ and $\{b\}$ by adding an appropriate context, for example $R_{3}=(\emptyset,\{b>a \stackrel{1}{\leftarrow}\})$. We obtain $\{b\}>^{P_{3} \cup R_{3}}\{a\}$, thus $\pi\left(P_{3} \cup R_{3}\right)=\{\{b\}\}$.

It is important to note that, in order to achieve this effect, the context must contain preference rules of lower ranks than the preference rules that originally ordered $I$ and $J$, as that original ordering has to be overridden. This also means that the technique is not applicable for preference rules of rank 1. In general, for any pair of strictly comparable interpretations $I, J$, where the comparison stems from preference rules of rank $>1$, adding any context consisting of preference rules of lower rank that reverse comparability of $I$ and $J$ results in the reversed strict order. As in Example 3, the context must make use of particular ranks.

These three effects may be exploited in order to construct examples of problems that are not strongly sel-equivalent and suggest necessary conditions for strong sel-equivalence. The first effect can turn preferred outcomes into non-preferred, while the second and third can turn non-preferred outcomes into preferred ones. The second and third effects imply conditions that are more specialized (context that needs rules of particular ranks).

Example 5 Consider $P_{4}=\left(T_{1},\{a>b \stackrel{2}{\leftarrow}\}\right)$ and $Q_{4}=\left(T_{1},\{a>b \stackrel{3}{\leftarrow}\}\right)$, where $T_{1}$ is a theory admitting exactly two outcomes $\{a\}$ and $\{b\}$. We have $\{a\}>^{P_{4}}\{b\},\{a\}>^{Q_{4}}\{b\}$, and $\pi\left(P_{4}\right)=\pi\left(Q_{4}\right)=\{\{a\}\}$. The two problems are therefore equivalent. However, there is 
a discrepancy with respect to the ranks of the preference rules, which we can take advantage of in order to show that the programs are not strongly sel-equivalent.

Let us consider the context $R_{4}=(\emptyset,\{b>a \stackrel{3}{\leftarrow}\})$. This context exploits the second effect mentioned above and makes $\{a\}$ and $\{b\}$ incomparable with respect to $Q_{4}$ extended with $R_{4}\left(\{a\} \ngtr^{Q_{4} \cup R_{4}}\{b\}\right.$ and $\left.\{b\} \ngtr^{Q_{4} \cup R_{4}}\{a\}\right)$ thus turning also $\{b\}$ into a preferred outcome $\pi\left(Q_{4} \cup R_{4}\right)=\{\{a\},\{b\}\}$. On the other hand, the new preference rule has no effect on $P_{4}$, as its rank is weaker than that of the only preference rule of $P_{4}$, hence $\pi\left(P_{4} \cup R_{4}\right)=\{\{a\}\}$, and therefore $P_{4} \not \equiv^{s,[3, i]} Q_{4}$ for all $i \geq 3$.

Analyzing this example, we can observe that what the context $R_{4}$ exploits is a difference in the preferred outcomes when considering only preference rules of rank lower than 3. Indeed, $\pi\left(\left(P_{4}\right)_{<3}\right)=\{\{a\}\}$ and $\pi\left(\left(Q_{4}\right)_{<3}\right)=\{\{a\},\{b\}\}$.

Example 6 Next, let us consider $P_{5}=P_{4}=\left(T_{1},\{a>b \stackrel{2}{\leftarrow}\}\right)$ and $Q_{5}=\left(T_{1},\{a>b \stackrel{4}{\leftarrow}\}\right)$, where $T_{1}$ is a theory admitting exactly two outcomes $\{a\}$ and $\{b\}$. We have $\{a\}>^{P_{5}}\{b\}$, $\{a\}>^{Q_{5}}\{b\}$, and $\pi\left(P_{5}\right)=\pi\left(Q_{5}\right)=\{\{a\}\}$. Also here, we can observe that $\pi\left(\left(P_{5}\right)_{<3}\right)=$ $\{\{a\}\}$ and $\pi\left(\left(Q_{5}\right)_{<3}\right)=\{\{a\},\{b\}\}$. The only difference to Example 5 is that the preference rule in $Q_{5}$ is of rank 4 .

Also here it is possible to construct a context that witnesses that $P_{5}$ is not strongly selequivalent to $Q_{5}$, using only preference rules of rank 3: $R_{5}=(\emptyset,\{a>b \stackrel{3}{\leftarrow}, b>a \stackrel{3}{\leftarrow}\})$. Here, we directly add conflicting preference rules that override the preference rule in $Q_{5}$ and are overridden by the preference rule in $P_{5}$. So, also here we get $\pi\left(P_{5} \cup R_{5}\right)=\{\{a\}\}$ and $\pi\left(Q_{5} \cup R_{5}\right)=\{\{a\},\{b\}\}$, and $P_{5} \not \equiv^{s,[3, i]} Q_{5}$ for all $i \geq 3$.

Examples 5 and 6 motivate condition (1) of Theorem 6 . Moreover, it is also rather easy to see that any counterexample to $P \equiv \equiv^{s,[i, j]} Q$ will involve only outcomes from $\pi\left(P_{<i}\right)=$ $\pi\left(Q_{<i}\right)$, as no selector context in the rank interval $[i, j]$ can make other outcomes preferred. However, from a different point of view, condition (1) of Theorem 6 is also fairly weak, as it does not cover some easy cases of strong sel-non-equivalence, as shown in the following example.

Example 7 Let us define $P_{6}=P_{4}=\left(T_{1},\{a>b \stackrel{2}{\leftarrow}\}\right)$ and $Q_{6}=\left(T_{1},\{b>a \stackrel{2}{\leftarrow}\}\right)$, where $T_{1}$ is a theory admitting exactly two outcomes $\{a\}$ and $\{b\}$. We have $\{a\}>^{P_{6}}\{b\},\{b\}>^{Q_{6}}\{a\}$, and $\pi\left(P_{6}\right)=\{\{a\}\} \neq \pi\left(Q_{6}\right)=\{\{b\}\}$. So $P_{6}$ and $Q_{6}$ are not even equivalent, hence also $P_{6} \not \equiv^{s,[i, j]} Q_{6}$ for any rank interval $[i, j]$. However, $\pi\left(\left(P_{6}\right)_{<2}\right)=\{\{a\},\{b\}\}$ and $\pi\left(\left(Q_{6}\right)_{<2}\right)=$ $\{\{a\},\{b\}\}$. So condition (1) of Theorem 6 is satisfied for rank intervals $[2, j]$.

Condition (2) of Theorem 6 covers cases like the one in Example 7. This example is rather simple, as it does not even require a context in order to create a witness for strong sel-non-equivalence, while in general one has to create a context in order to make certain outcomes preferred. What remains to be considered are cases in which there is a discrepancy stemming from preference rules inside the context rank interval.

Example 8 Let $P_{7}=\left(T_{1},\{a>b \stackrel{2}{\leftarrow}\}\right)$ and $Q_{7}=\left(T_{1},\{a>b \stackrel{3}{\leftarrow}\}\right)$, where $T_{1}$ is a theory admitting exactly two outcomes $\{a\}$ and $\{b\}$ (this is the pair of problems $P_{4}$ and $Q_{4}$ from Example 5). Consider now the context $R_{7}=(\emptyset,\{b>a \stackrel{2}{\leftarrow}\})$. Unlike $R_{4}$ in Example 5, this 
rule has rank 2. The context makes $\{a\}$ and $\{b\}$ incomparable with respect to the extended $P_{7}\left(\{a\} \ngtr^{P_{7} \cup R_{7}}\{b\}\right.$ and $\left.\{b\} \ngtr^{P_{7} \cup R_{7}}\{a\}\right)$, thus turning $\{b\}$ into a preferred outcome, and keeping $\{a\}$ as such. Therefore, $\pi\left(P_{7} \cup R_{7}\right)=\{\{a\},\{b\}\}$. On the other hand, the new preference rule overrides the one of $Q_{7}$, turning $\{b\}$ into a preferred outcome and making $\{a\}$ non-preferred, $\pi\left(Q_{7} \cup R_{7}\right)=\{\{b\}\}$. Therefore $P_{7} \not \equiv^{s,[2, i]} Q_{7}$ for all $i \geq 2$.

Unlike in Example 5, $\pi\left(\left(P_{7}\right)_{<2}\right)=\pi\left(\left(Q_{7}\right)_{<2}\right)=\{\{a\},\{b\}\}$, so there is a different reason that allows for this counterexample. Here, we observe that $\operatorname{diff}^{P_{7}}(\{a\},\{b\})=2 \neq$ $\operatorname{diff}^{Q_{7}}(a, b)=3$, which allows for adding an appropriate preference rule of rank 2. It is important that we had to add a rule of one of the two differing ranks. Indeed, there is no context comprising only rules of rank one that can serve as a counterexample to strong sel-equivalence, and indeed $P_{7} \equiv^{s,[1,1]} Q_{7}$.

This finally motivates condition (3) of Theorem 6: if there are two outcomes (as discussed earlier, we can restrict ourselves to outcomes in $\pi\left(P_{<i}\right)=\pi\left(Q_{<i}\right)$ for a context rank interval $[i, j])$ which differ on ranks such that one of the ranks is inside the rank interval, we can use constructions as in Example 8 in order to obtain a counterexample to strong sel-equivalence. We can show that the three conditions of Theorem 6 indeed do characterize strong selequivalence.

Theorem 6 For all ranked optimization problems $P$ and $Q$, and every rank interval $[i, j]$, $P \equiv{ }^{s,[i, j]} Q$ if and only if the following conditions hold:

1. $\pi\left(P_{<i}\right)=\pi\left(Q_{<i}\right)$

2. $>_{\pi\left(P_{<i}\right)}^{P}=>_{\pi\left(Q_{<i}\right)}^{Q}$

3. for every $I, J \in \pi\left(P_{<i}\right)$ such that $i<\operatorname{diff}^{P}(I, J)$ or $i<\operatorname{diff}^{Q}(I, J)$, $\operatorname{diff}^{P}(I, J)=$ $\operatorname{diff}^{Q}(I, J)$ or both $\operatorname{diff}^{P}(I, J)>j$ and $\operatorname{diff}^{Q}(I, J)>j$.

The proof of this result is quite involved and requires several auxiliary properties. We provide it in the appendix (together with proofs of our other main results).

Next, we discuss some special cases of the characterization in Theorem 6. First, we consider the case $i=1$, which allows for a simplification of Theorem 6 .

Corollary 7 For all ranked optimization problems $P$ and $Q$, and every rank interval $[1, j]$, $P \equiv s,[1, j] Q$ if and only if the following conditions hold:

1. $\mu(P)=\mu(Q)$

2. $>_{\mu(P)}^{P}=>_{\mu(Q)}^{Q}$

3. for every $I, J \in \mu(P)$ diff $^{P}(I, J)=\operatorname{diff}^{Q}(I, J)$ or both $\operatorname{diff}^{P}(I, J)>j$ and $\operatorname{diff}^{Q}(I, J)>$ $j$.

Proof. Starting from Theorem 6, we note that the selectors of $P_{<1}$ and $Q_{<1}$ are empty and hence $\pi\left(P_{<1}\right)=\mu(P)$ and $\pi\left(Q_{<1}\right)=\mu(Q)$. Moreover, if the precondition $i<\operatorname{diff}^{P}(I, J)$ or $i<\operatorname{diff}^{Q}(I, J)$ in condition (3) of Theorem 6 is not satisfied for $i=1$ and a pair $I, J \in \mu(P)$, then one of $\operatorname{diff}^{P}(I, J)=1$ and $\operatorname{diff}^{Q}(I, J)=1$ holds, and together with 
$\operatorname{diff}^{P}(I, J)=\operatorname{diff}^{Q}(I, J)$ the consequent is satisfied in that case as well, which allows for omitting the precondition.

If in addition $j=\infty$, we obtain the case of rank-unrestricted selector contexts, and condition (3) can be simplified once more, since $\operatorname{diff}^{P}(I, J)>j$ and $\operatorname{diff}^{Q}(I, J)>j$ never hold for $j=\infty$.

Corollary 8 For all optimization problems $P$ and $Q, P \equiv{ }^{s} Q$ (equivalently, $P \equiv{ }^{s, \geq 1} Q$ or $P \equiv s,[1, \infty] Q)$ if and only if the following conditions hold:

1. $\mu(P)=\mu(Q)$

2. $>_{\mu(P)}^{P}=>_{\mu(Q)}^{Q}$

3. for every $I, J \in \mu(P)$, $\operatorname{diff}^{P}(I, J)=\operatorname{diff}^{Q}(I, J)$.

Next, we note that if an optimization problem $P$ is simple (all rules are of rank 1), then $\operatorname{diff}^{P}(I, J)>1$ if and only if $\operatorname{diff}^{P}(I, J)=\infty$, which is equivalent to $I \approx^{P} J$. This observation leads to the following characterization of strong sel-equivalence of simple optimization problems.

Corollary 9 For all simple optimization problems $P$ and $Q$, the following statements are equivalent:

(a) $P \equiv^{s} Q$ (equivalently, $P \equiv^{s,[1, \infty]} Q$ )

(b) $P \equiv^{s,=1} Q$ (equivalently, $P \equiv^{s,[1,1]} Q$ )

(c) $\mu(P)=\mu(Q)$ and $\geq_{\mu(P)}^{P}=\geq_{\mu(Q)}^{Q}$.

Proof. The implication (a) $\Rightarrow(\mathrm{b})$ is evident from the definitions.

(b) $\Rightarrow$ (c) From Corollary 7 , with $j=1$, we obtain $\mu(P)=\mu(Q)$. The condition $\geq_{\mu(P)}^{P}=\geq_{\mu(Q)}^{Q}$ follows from conditions (2) and (3) of that corollary. Indeed, let us consider $I, J \in \mu(P)$ such that $I \geq^{P} J$ and distinguish two cases. If (i) $\operatorname{diff}^{P}(I, J)=1$ then $I>^{P} J$ and by condition (2) of Corollary 7, also $I>^{Q} J$, implying $I \geq^{Q} J$. If (ii) $\operatorname{diff}^{P}(I, J)>1$ then by condition (3) of Corollary $7, \operatorname{diff}^{Q}(I, J)>1$. Since $P, Q$ are simple, $I \approx^{Q} J$, and consequently $I \geq^{Q} J$. By symmetry, we also have that $I \geq^{Q} J$ implies $I \geq^{P} J$. Thus, $\geq_{\mu(P)}^{P}=\geq_{\mu(Q)}^{Q}$.

(c) $\Rightarrow$ (a) From (c) it follows by Lemma 1 that $>_{\mu(P)}^{P}=>_{\mu(Q)}^{Q}$ and $\approx_{\mu(P)}^{P}=\approx_{\mu(Q)}^{Q}$. Thus, conditions (1) and (2) of Corollary 8 follow. To prove condition (3), let us first assume $\operatorname{diff}^{P}(I, J)>1$ for $I, J \in \mu(P)$. It follows that $\operatorname{diff}^{P}(I, J)=\infty$ and thus $I \approx_{\mu(P)}^{P} J$. Since $\approx_{\mu(P)}^{P}=\approx_{\mu(Q)}^{Q}$, we get $I \approx_{\mu(Q)}^{Q} J$ and thus $\operatorname{diff}^{Q}(I, J)=\infty$. Hence $\operatorname{diff}^{P}(I, J)=\operatorname{diff}^{Q}(I, J)$. For $\operatorname{diff}^{Q}(I, J)>1$ we reason analogously. In the last remaining case, $\operatorname{diff}^{P}(I, J)=1$ and $\operatorname{diff}^{Q}(I, J)=1$. Thus, we directly obtain $\operatorname{diff}^{P}(I, J)=\operatorname{diff}^{Q}(I, J)$. By Corollary $8, P \equiv^{s} Q$ follows. 
Corollary 9 shows, in particular, that for simple problems there is no difference between the relations $\equiv^{s, \geq 1}$ and $\equiv^{s,=1}$. This property reflects the role of preference rules of rank 2 and higher. They allow us to break ties among optimal outcomes, as defined by preference rules of rank 1 . Thus, they can eliminate some of these outcomes from the family of optimal ones, but they cannot introduce new optimal outcomes. Therefore, they do not affect strong sel-equivalence of simple problems. This property has the following generalization to ranked optimization problems.

Corollary 10 Let $P$ and $Q$ be ranked optimization problems and let $k$ be the maximum rank of a preference rule in $P \cup Q$. Then the relations $\equiv^{s, \geq k}$ (equivalently, $\equiv^{s,[k, \infty]}$ ) and $\equiv^{s,=k}$ (equivalently, $\equiv^{s,[k, k]}$ ) coincide.

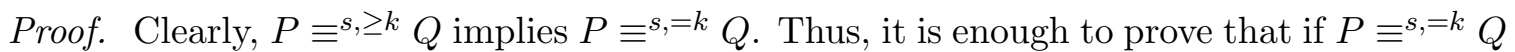
then $P \equiv{ }^{s, \geq k} Q$. Using the characterization of Theorem 6 , we observe that conditions (1) and (2) for $P \equiv^{s,=k} Q$ and $P \equiv^{s, \geq k} Q$ are the same. Since $P \equiv^{s,=k} Q$, we have that for every $I, J \in \pi\left(P_{<k}\right)$ such that $k<\operatorname{diff}^{P}(I, J)$ or $k<\operatorname{diff}^{Q}(I, J) \operatorname{diff}^{P}(I, J)=\operatorname{diff}^{Q}(I, J)$ or both $\operatorname{diff}^{P}(I, J)>k$ and $\operatorname{diff}^{Q}(I, J)>k$. Let us consider $I, J \in \pi\left(P_{<k}\right)$ such that $\operatorname{diff}^{P}(I, J)>k$. It follows that $\operatorname{diff}^{Q}(I, J)>k$. Since $k$ is the maximum rank of a preference rule in $P$ or $Q, \operatorname{diff}^{P}(I, J)=\infty$ and $\operatorname{diff}^{Q}(I, J)=\infty$. Thus, $\operatorname{diff}^{P}(I, J)=\operatorname{diff}^{Q}(I, J)$. The case $\operatorname{diff}^{Q}(I, J)>k$ is similar and we obtain that for every $I, J \in \pi\left(P_{<k}\right)$ such that $k<\operatorname{diff}^{P}(I, J)$ or $k<\operatorname{diff}^{Q}(I, J)$, diff ${ }^{P}(I, J)=\operatorname{diff}^{Q}(I, J)$. That property implies condi-

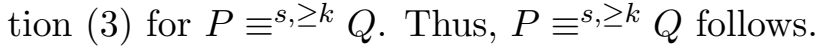

Our observation on the role of preference rules with ranks higher than ranks of rules in $P$ or $Q$ also implies that $P$ and $Q$ are strongly sel-equivalent relative to selectors consisting exclusively of such rules if and only if $P$ and $Q$ are equivalent (have the same optimal outcomes), and if optimal outcomes that "tie" in $P$ also "tie" in $Q$ and conversely. Formally, we have the following result.

Corollary 11 Let $P$ and $Q$ be ranked optimization problems and let $k$ be the maximum rank of a preference rule in $P \cup Q$. Then $P \equiv^{s, \geq k+1} Q$ if and only if $\pi(P)=\pi(Q)$ and $\approx_{\pi(P)}^{P}=\approx_{\pi(Q)}^{Q}$

Proof. Clearly, $P_{<k+1}=P$ and $Q_{<k+1}=Q$ and so, $\pi\left(P_{<k+1}\right)=\pi(P)$ and $\pi\left(Q_{<k+1}\right)=$ $\pi(Q)$. Thus, the "only-if" part follows by Theorem 6 (condition (1) of that theorem reduces to $\pi(P)=\pi(Q)$ and condition (3) implies $\left.\approx_{\pi(P)}^{P}=\approx_{\pi(Q)}^{Q}\right)$. To prove the "if" part, we note that condition (1) of Theorem 6 holds by the assumption. Moreover, the relations $>_{\pi(P)}^{P}$ and $>_{\pi(Q)}^{Q}$ are empty and so, they coincide. Thus, condition (2) of Theorem 6 holds. Finally, if $I, J \in \pi(P)$, and $\operatorname{diff}^{P}(I, J)>k+1$, then $\operatorname{diff}^{P}(I, J)=\infty$ and so, $I \approx^{P} J$. By the assumption, $I \approx^{Q} J$, that is, $\operatorname{diff}^{Q}(I, J)=\infty=\operatorname{diff}^{P}(I, J)$. The case when $\operatorname{diff}^{Q}(I, J)>k+1$ is similar. Thus, condition (3) of Theorem 6 holds, too, and $P \equiv{ }^{s, \geq k+1} Q$ follows.

Lastly, we give some simple examples illustrating how our results can be used to "safely" modify or simplify optimization problems, that is rewrite one into another strongly selequivalent one. 
Example 9 Let $P=(T, S)$, where $T=\{a \vee b \vee c, \neg(a \wedge b), \neg(a \wedge c), \neg(b \wedge c)\}$ and $S=\{a>$ $c \leftarrow, b>c \leftarrow\}$, and $P^{\prime}=\left(T, S^{\prime}\right)$, where $S^{\prime}=\{a \vee b>c \leftarrow\}$. Regarding these problems as CO problems, we have that $\mu(P)=\mu\left(P^{\prime}\right)=\{\{a\},\{b\},\{c\}\}$. Moreover, it is evident that $\geq_{\mu(P)}^{P}=\geq_{\mu\left(P^{\prime}\right)}^{P^{\prime}}$. Thus, by Corollary 9, $P$ and $P^{\prime}$ are strongly sel-equivalent. In other words, we can faithfully replace rules $a>c \leftarrow, b>c \leftarrow$ in the selector of any optimization problem with generator $T$ by the single rule $a \vee b>c \leftarrow$.

For an example of a more general principle, we note that removing preference rules with only one formula in the head yields a problem that is strongly sel-equivalent.

Corollary 12 Let $P$ and $Q$ be two $C O$ or $A S O$ problems such that $P^{g}=Q^{g}$ and $Q^{s}$ is obtained from $P^{s}$ by removing all preference rules with only one formula in the head (i.e., rules $r$ for which $|h d(r)|=1)$. Then $P$ and $Q$ are strongly sel-equivalent.

Proof. Conditions (1)-(3) of Theorem 6 all follow from an observation that for every interpretation $I$ and every preference rule $r$ with $|h d(r)|=1, v_{I}(r)=1$.

\section{Strong Gen-Equivalence}

We now focus on the case of strong gen-equivalence. The semantics of generators makes a difference here but the difference concerns only the fact that under the two semantics we consider, the concepts of strong equivalence are different. Other aspects of the characterizations are the same. Specifically, generators have to be strongly equivalent relative to the selected semantics. Indeed, as the following example shows, if the generators are not strongly equivalent, one can extend them uniformly so that after the extension one problem has a single outcome, which is then trivially an optimal one, too, while the other one has no outcomes and so, no optimal ones.

Example 10 Consider a CO problem $P_{8}=\left(T_{8}, S_{8}\right)$, where $T_{8}=\{a \leftrightarrow \neg b\}$ and $S_{8}=\{a>$ $b \leftarrow\}$. There are two outcomes here, $\{a\}$ and $\{b\}$, that is, $\mu\left(P_{8}\right)=\{\{a\},\{b\}\}$. Let $r$ be the only preference rule in $S_{8}$. Clearly, $v_{\{a\}}(r)=1$ and $v_{\{b\}}(r)=2$. Thus, $\{a\}>^{P_{8}}\{b\}$ and so, $\pi\left(P_{8}\right)=\{\{a\}\}$.

In addition, let $Q_{8}=\left(T_{8}^{\prime}, S_{8}\right)$ be a $C O$ problem, where $T_{8}^{\prime}=\{a \wedge \neg b\}$ and $S_{8}$ is as above. Then, $\mu\left(Q_{8}\right)=\{\{a\}\}$ and, trivially, $\pi\left(Q_{8}\right)=\{\{a\}\}$. It follows that $P_{8}$ and $Q_{8}$ are equivalent, as they specify the same optimal outcomes. However, they are not strongly gen-equivalent (and so, also not strongly equivalent). Indeed, let $R_{8}=(\{\neg a\}, \emptyset)$. Then $\mu\left(P_{8} \cup R_{8}\right)=\{\{b\}\}$ and so, $\pi\left(P_{8} \cup R_{8}\right)=\{\{b\}\}$. On the other hand, $\mu\left(Q_{8} \cup R_{8}\right)=\emptyset$ and, therefore, $\pi\left(Q_{8} \cup R_{8}\right)=\emptyset$.

Moreover, the preference relation $>$ defined by the selectors of the problems considered must coincide.

Example 11 Let $P_{9}=\left(T_{9}, S_{9}\right)$ be a CO problem, where $T_{9}=\{a \vee b \vee c, \neg(a \wedge b), \neg(a \wedge$ $c), \neg(b \wedge c)\}$ and $S_{9}=\{a>b \leftarrow, a>c \leftarrow\}$. We have $\mu\left(P_{9}\right)=\{\{a\},\{b\},\{c\}\}$, and $\{a\}>^{P_{9}}\{b\},\{a\}>^{P_{9}}\{c\}$, and $\{b\}$ and $\{c\}$ are incomparable. Thus, $\pi\left(P_{9}\right)=\{\{a\}\}$. Let 
now $Q_{9}=\left(T_{9}, S_{9}^{\prime}\right)$ be a CO problem, where $S_{9}^{\prime}=\{a>b>c \leftarrow\}$. Clearly, $\mu\left(Q_{9}\right)=\mu\left(P_{9}\right)=$ $\{\{a\},\{b\},\{c\}\}$. Moreover, $\{a\}>^{Q_{9}}\{b\}>^{Q_{9}}\{c\}$. Thus, $\pi\left(Q_{9}\right)=\{\{a\}\}$ and so, $P_{9}$ and $Q_{9}$ are equivalent. However, they are not strongly (gen-)equivalent. Indeed, let $R_{9}=(\{\neg a\}, \emptyset)$. Then, $\pi\left(P_{9} \cup R_{9}\right)=\{\{b\},\{c\}\}$ but $\pi\left(P_{9} \cup R_{9}\right)=\{\{b\}\}$.

The main insight here is that differences in the preference relation may be "hidden" by preferred outcomes but, if they are present, they can be exposed by eliminating the preferred outcomes that obscure them with the appropriately selected generator context.

All of these considerations apply to both the CO and ASO problems, and therefore we formulate a single theorem that handles both types of problems.

Theorem 13 For all $C O$ (ASO, respectively) problems $P$ and $Q, P \equiv_{g} Q$ if and only if $P^{g}$ and $Q^{g}$ are strongly equivalent (that is, $\operatorname{Mod}\left(P^{g}\right)=\operatorname{Mod}\left(Q^{g}\right)$ for CO problems, and $\operatorname{Mod}_{H T}\left(P^{g}\right)=\operatorname{Mod}_{H T}\left(Q^{g}\right)$ for ASO problems) and $>_{\operatorname{Mod}\left(P^{g}\right)}^{P}=>_{M o d\left(Q^{g}\right)}^{Q}$.

In view of Examples 10 and 11, the result is not unexpected. The two examples demonstrated that the conditions of the characterization cannot, in general, be weakened.

From Corollary 8 and Theorem 13, it follows that strong sel-equivalence of CO problems is a stronger property than their strong gen-equivalence.

Corollary 14 For all $C O$ problems $P$ and $Q, P \equiv{ }^{s} Q$ implies $P \equiv_{g} Q$.

In general the implication in Corollary 14 cannot be reversed, as shown in the following example.

Example 12 Let us consider problems $P_{10}=\left(T_{10}, S_{10}\right)$ and $Q_{10}=\left(T_{10}, \emptyset\right)$, where $T_{10}=$ $\{a \leftrightarrow \neg b\}$ and $S_{10}=\{a>b \leftarrow, b>a \leftarrow\}$. We have $\mu\left(P_{10}\right)=\mu\left(Q_{10}\right)=\{\{a\},\{b\}\}$. Moreover, $\{a\} \nsupseteq^{P_{10}}\{b\}$ and $\{b\} \nsupseteq^{P_{10}}\{a\}$. Thus, $\pi\left(P_{10}\right)=\{\{a\},\{b\}\}$. Since $Q_{10}^{s}=$ $\emptyset$, we also have (trivially) that $\{a\} \approx^{Q_{10}}\{b\}$. Thus, $\pi\left(Q_{10}\right)=\{\{a\},\{b\}\}$, too, and the problems $P_{10}$ and $Q_{10}$ are equivalent. They are not strongly sel-equivalent, though. Let $R_{10}=(\emptyset,\{a>b \leftarrow\})$. Then, $P_{10} \cup R_{10}=P_{10}$ and so, $\pi\left(P_{10} \cup R_{10}\right)=\{\{a\},\{b\}\}$. On the other hand, $\{a\}>Q_{10} \cup R_{10}\{b\}$. Thus, $\pi\left(Q_{10} \cup R_{10}\right)=\{\{a\}\}$.

However, by virtue of Theorem 13, they are strongly gen-equivalent. Indeed, trivially $\operatorname{Mod}\left(P_{10}^{g}\right)=\operatorname{Mod}\left(Q_{10}^{g}\right)$ and, writing $M$ for $\operatorname{Mod}\left(P_{10}^{g}\right)=\operatorname{Mod}\left(Q_{10}^{g}\right)$, the relations $>_{M}^{P_{10}}$ and $>_{M}^{Q_{10}}$ are both empty and therefore equal.

The relation between strong sel-equivalence and strong gen-equivalence of ASO problem is more complex. In general, neither property implies the other even if both problems $P$ and $Q$ are assumed to be simple. It is so because $P \equiv^{s} Q$ if and only if $A S\left(P^{g}\right)=$ $A S\left(Q^{g}\right)$ and $\geq_{A S\left(P^{g}\right)}^{P}=\geq_{A S\left(Q^{g}\right)}^{Q}$ (Corollary 9), and $P \equiv_{g} Q$ if and only of $\operatorname{Mod}_{H T}\left(P^{g}\right)=$ $\operatorname{Mod}_{H T}\left(Q^{g}\right)$ and $>_{M o d\left(P^{g}\right)}^{P}=>_{M o d}^{Q}\left(Q^{g}\right)$ (Theorem 13). Now, $A S\left(P^{g}\right)=A S\left(Q^{g}\right)$ (regular equivalence of programs) does not imply $\operatorname{Mod}_{H T}\left(P^{g}\right)=\operatorname{Mod}_{H T}\left(Q^{g}\right)$ (strong equivalence) and $>_{M o d\left(P^{g}\right)}^{P}=>_{M o d\left(Q^{g}\right)}^{Q}$ does not imply $\geq_{A S\left(P^{g}\right)}^{P}=\geq_{A S\left(Q^{g}\right)}^{Q}$.

We conclude the section with one more corollary concerning strong gen-equivalence of problems with empty selectors. 
Corollary 15 For all $C O$ (ASO, respectively) problems $P$ and $Q$ such that $P^{s}=Q^{s}=\emptyset$, $P \equiv \equiv_{g} Q$ if and only if $P^{g}$ and $Q^{g}$ are strongly equivalent under the respective semantics (that is, $\operatorname{Mod}\left(P^{g}\right)=\operatorname{Mod}\left(Q^{g}\right)$ for $C O$ problems, and $\operatorname{Mod}_{H T}\left(P^{g}\right)=\operatorname{Mod}_{H T}\left(Q^{g}\right)$ for $A S O$ problems).

The result is evident from the definitions. However, it is also an immediate consequence of Theorem 13. Indeed, when optimization problems $P$ and $Q$ have empty selectors, the condition $>_{\operatorname{Mod}\left(P^{g}\right)}^{P}=>_{\operatorname{Mod}\left(Q^{g}\right)}^{Q}$ is equivalent to $\operatorname{Mod}\left(P^{g}\right)=\operatorname{Mod}\left(Q^{g}\right)$, which is a consequence of strong equivalence of $P^{g}$ and $Q^{g}$. Thus, for problems with empty selectors the "right hand" of the equivalence in the assertion of Theorem 13 reduces to just strong equivalence of the generators.

\section{Strong Equivalence - the Combined Case}

Finally, we consider the relation $\equiv_{g}^{s}$, which results from considering contexts that combine both generators and selectors. Since generators may vary here, as in the previous section, the semantics of generators matters. But, as in the previous section, the difference boils down to different characterizations of strong equivalence of generators.

We start with a result characterizing strong equivalence of $\mathrm{CO}$ and ASO problems relative to combined contexts (both generators and selectors possibly non-empty) with selectors consisting of rules of rank at least $i$ and at most $j$, respectively.

Theorem 16 For all ranked $C O$ (ASO, respectively) problems $P$ and $Q$, and every rank interval $[i, j], P \equiv_{g}^{s,[i, j]} Q$ if and only if the following conditions hold:

1. $P^{g}$ and $Q^{g}$ are strongly equivalent (that is, $\operatorname{Mod}\left(P^{g}\right)=\operatorname{Mod}\left(Q^{g}\right)$ for CO problems, and $\operatorname{Mod}_{H T}\left(P^{g}\right)=\operatorname{Mod}_{H T}\left(Q^{g}\right)$ for ASO problems)

2. $>_{\operatorname{Mod}\left(P^{g}\right)}^{P}=>_{\operatorname{Mod}\left(Q^{g}\right)}^{Q}$

3. for every $I, J \in \operatorname{Mod}\left(P^{g}\right)$ such that $i<\operatorname{diff}^{P}(I, J)$ or $i<\operatorname{diff}^{Q}(I, J)$, $\operatorname{diff}^{P}(I, J)=$ $\operatorname{diff}^{Q}(I, J)$ or both $\operatorname{diff}^{P}(I, J)>j$ and $\operatorname{diff}^{Q}(I, J)>j$

4. $>_{\operatorname{Mod}\left(P^{g}\right)}^{P_{<i}}=>_{\operatorname{Mod}\left(Q^{g}\right)}^{Q_{<i}}$.

The corresponding characterizations for CO and ASO problems differ only in their respective conditions (1), which now reflect different conditions guaranteeing strong equivalence of generators under the classical and answer-set semantics. Moreover, the four conditions of Theorem 16 can be obtained by suitably combining and extending the conditions of Theorem 6 and Theorem 13. First, as combined strong equivalence implies strong genequivalence, condition (1) is taken from Theorem 13. Second, we modify conditions (2) and (3) from Theorem 6 replacing $\pi\left(P_{<i}\right)$ with $\operatorname{Mod}\left(P^{g}\right)$ (and accordingly $\pi\left(Q_{<i}\right)$ with $\left.\operatorname{Mod}\left(Q^{g}\right)\right)$, as each classical model of $P^{g}$ can give rise to an optimal classical or equilibrium one upon the addition of a context, an aspect also already visible in Theorem 13.

Finally, we have to add a new condition stating that the relations $>^{P<i}$ and $>^{Q_{<i}}$ coincide on the sets of models of $P^{g}$ and $Q^{g}$. When generators are allowed to be extended, one can make any two of their models to be the only outcomes after the extension. If the two 
outcomes, say $I$ and $J$, are related differently by the corresponding strict relations induced

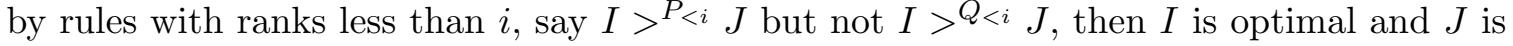
not optimal in the problem extending $P$ no matter what preference rules of ranks from the interval $[i, j]$ we use (rules of rank $i$ and higher have no effect on how $I$ and $J$ are ordered). On the other hand, if $I$ and $J$ are incomparable by $>^{Q_{<i}}$, they remain incomparable after $Q$ is extended. If $I$ and $J$ are equally good with respect to rules of rank $<i$, they can be rendered incomparable by means of preference rules of rank $i$. In each case, $J$ is optimal after $Q$ is extended. Finally, if $J>^{Q_{<i}} I$, then $J$ remains optimal no matter what preference rules of ranks $i$ and higher we add. It follows that if the two relations $>^{P<i}$ and $>^{Q_{<i}}$ are different, we cannot have $P \equiv_{g}^{s,[i, j]} Q$ and the condition (4) is necessary.

As in the previous section, the case when the selectors of $P$ and $Q$ are empty reduces to strong gen-equivalence of the generators.

Corollary 17 For all $C O$ (ASO, respectively) problems $P$ and $Q$ such that $P^{s}=Q^{s}=$ $\emptyset$, and for every rank interval $[i, j], P \equiv_{g}^{s,[i, j]} Q$ if and only if $P^{g}$ and $Q^{g}$ are strongly equivalent under the respective semantics (that is, $\operatorname{Mod}\left(P^{g}\right)=\operatorname{Mod}\left(Q^{g}\right)$ for CO problems, and $\operatorname{Mod}_{H T}\left(P^{g}\right)=\operatorname{Mod}_{H T}\left(Q^{g}\right)$ for ASO problems).

The result is a simple consequence of Theorem 16. The condition (1) in that theorem implies that $\operatorname{Mod}\left(P^{g}\right)=\operatorname{Mod}\left(Q^{g}\right)$ and so, since the selectors of $P$ and $Q$ are empty, all the remaining conditions become trivially true.

We conclude this section with observations concerning the relation $\equiv_{g}^{s}$ for both $\mathrm{CO}$ and ASO problems. The contexts relevant here may contain preference rules of arbitrary ranks. We start with the case of $\mathrm{CO}$ problems, where the results are stronger. While they can be derived from the general theorems above, we will present here arguments relying on results from previous sections, which is possible since for $\mathrm{CO}$ problems equivalence and strong equivalence of generators coincide.

We saw in the last section that for CO problems $\equiv^{s}$ is a strictly stronger relation than $\equiv_{g}$. In fact, for CO problems, $\equiv^{s}$ coincides with the general relation $\equiv_{g}^{s}$.

Theorem 18 For all $C O$ problems $P$ and $Q, P \equiv_{g}^{s} Q$ if and only if $P \equiv^{s} Q$.

Proof. The "only-if" implication is evident. To prove the converse implication, we will use Proposition 4, which reduces checking for strong equivalence to checking for strong sel-equivalence. Let $R \in \mathcal{L}_{\mathcal{U}}^{g}$ be a generator problem. Since $P \equiv^{s} Q$, from Corollary 8 we have $\operatorname{Mod}\left(P^{g}\right)=\operatorname{Mod}\left(Q^{g}\right)$. Consequently, $\operatorname{Mod}\left((P \cup R)^{g}\right)=\operatorname{Mod}\left((Q \cup R)^{g}\right)$. Writing $M$ for $\operatorname{Mod}\left(P^{g}\right)$ and $M^{\prime}$ for $\operatorname{Mod}\left((P \cup R)^{g}\right)$ we have $M^{\prime} \subseteq M$. Thus, also by Corollary 8, $>_{M^{\prime}}^{P \cup R}=>_{M^{\prime}}^{Q \cup R}$. Finally, condition (3) of Corollary 8 for $P$ and $Q$ implies condition (3) of that corollary for $P \cup R$ and $Q \cup R$ (as $R$ has no preference rules and $M^{\prime} \subseteq M$ ). It follows, again by Corollary 8, that $P \cup R \equiv^{s} Q \cup R$. Thus, by Proposition $4, P \equiv_{g}^{s} Q$.

In the case of simple $\mathrm{CO}$ problems and the ranked interval $[1,1]$, the argument above can be repeated using instead of Corollary 8 the equivalence of (b) and (c) from Corollary 9. In this way, one can show that for simple CO problems, the relations $\equiv_{g}^{s,=1}$ and $\equiv^{s,=1}$ coincide. Thus, by Corollary 9 (the equivalence of (a) and (b)) and Theorem 18, for simple CO problems all four relations $\equiv_{g}^{s}, \equiv_{g}^{s,=1}, \equiv^{s,=1}$, and $\equiv^{s}$ coincide and we obtain the following result. 
Corollary 19 For all simple $C O$ problems $P$ and $Q$, all properties $P \equiv_{g}^{s} Q, P \equiv_{g}^{s,=1} Q$, $P \equiv{ }^{s,=1} Q$ and $P \equiv^{s} Q$ are equivalent.

For simple ASO problems we still have that $\equiv_{g}^{s}$ and $\equiv_{g}^{s,=1}$ coincide but in general these notions are different from $\equiv^{s}$ and $\equiv^{s,=1}$ (cf. the subtle difference in condition (c) compared to Corollary 9).

Corollary 20 For all simple ASO problems $P$ and $Q$, the following conditions are equivalent

(a) $P \equiv_{g}^{s} Q$ (equivalently, $P \equiv_{g}^{s,[1, \infty]} Q$ )

(b) $P \equiv_{g}^{s,=1} Q$ (equivalently, $P \equiv_{g}^{s,[1,1]} Q$ )

(c) $\operatorname{Mod}_{H T}\left(P^{g}\right)=\operatorname{Mod}_{H T}\left(Q^{g}\right)$ and $\geq_{\operatorname{Mod}\left(P^{g}\right)}^{P}=\geq_{\operatorname{Mod}\left(Q^{g}\right)}^{Q}$.

Proof. The implication (a) $\Rightarrow(\mathrm{b})$ is evident.

Let us assume (b). By Theorem 16, we have $\operatorname{Mod}_{H T}\left(P^{g}\right)=\operatorname{Mod}_{H T}\left(Q^{g}\right)$. This identity implies $\operatorname{Mod}\left(P^{g}\right)=\operatorname{Mod}\left(Q^{g}\right)$. Let us assume that for some $I, J \in \operatorname{Mod}\left(P^{g}\right), I \geq_{M o d\left(P^{g}\right)}^{P} J$. If $I>_{M o d\left(P^{g}\right)}^{P} J$ then, by Theorem 16, $I>_{\operatorname{Mod}\left(Q^{g}\right)}^{Q} J$ and so, $I \geq_{\operatorname{Mod}\left(Q^{g}\right)}^{Q} J$. Otherwise, $I \approx^{P} J$ and so, $\operatorname{diff}^{P}(I, J)=\infty$. By Theorem 16 , diff ${ }^{Q}(I, J)>1$. Since $Q$ is simple, $\operatorname{diff}^{Q}(I, J)=\infty$. Thus, $I \approx^{Q} J$ and, also, $I \geq_{M o d\left(Q^{g}\right)}^{Q} J$. The converse implication follows by symmetry. Thus, (c) holds.

Finally, we assume (c) and prove (a). To this end, we show that conditions (1)-(4) of Theorem 16 hold. Directly from the assumptions, we have that condition (1) holds. Condition (2) follows from Lemma 1. Moreover, we also have that $\operatorname{Mod}\left(P^{g}\right)=\operatorname{Mod}\left(Q^{g}\right)$. To prove condition (3), let us assume that $I, J \in \operatorname{Mod}\left(P^{g}\right)$ and that $\operatorname{diff}^{P}(I, J)>1$. Since $P$ is simple, $I \approx^{P} J$. Thus, $I \approx^{Q} J$ and, consequently, $\operatorname{diff}^{P}(I, J)=\infty=\operatorname{diff}^{Q}(I, J)$. Finally, condition (4), i.e. $>_{M o d\left(P^{g}\right)}^{P_{<i}}=>_{\operatorname{Mod}\left(Q^{g}\right)}^{Q_{<i}}$, obviously holds in case $i=1$ and $\operatorname{Mod}\left(P^{g}\right)=\operatorname{Mod}\left(Q^{g}\right)$.

\section{Complexity}

In this section, we study the problems of deciding the various notions of strong equivalence. Typically the comparisons between sets of outcomes in the characterizations determine the respective complexity. We start with results concerning strong sel-equivalence.

Theorem 21 Given optimization problems $P$ and $Q$, deciding $P \equiv{ }^{s} Q$ is co-NP-complete in case of $C O$ problems and $\Pi_{2}^{P}$-complete in case of ASO problems.

Proof. [Sketch, a detailed argument is provided in Appendix B.] For membership, we focus on the complementary problem and consider pairs of interpretations $I, J$ that violate at least one of the conditions stated in Corollary 8. Clearly, if such a witness pair of interpretations exists, there is also such a witness pair that is built only from atoms that occur in the problems $P$ and $Q$. Once such a pair is guessed, it can be verified in polynomial time (for $\mathrm{CO}$ problems) or in polynomial time using an NP oracle (for ASO problems) that it indeed 
violates the conjunction of the three conditions from Corollary 8. The main observation is that model checking is polynomial for the classical semantics, but co-NP-complete for the equilibrium semantics (see Pearce, Tompits, \& Woltran, 2009, Thm. 8).

Hardness follows from considering the equivalence problem for optimizations problems with empty selectors, which is known to be co-NP-hard (for classical semantics) and $\Pi_{2}^{P}$ hard (for equilibrium semantics, see Pearce et al., 2009, Thm. 11).

For the ranked case, we observe an increase in complexity, which can be explained by the characterization given in Theorem 6 . Instead of outcome checking, this characterization involves optimal outcome checking, which is more difficult (unless the polynomial hierarchy collapses).

Theorem 22 Given optimization problems $P$ and $Q$ and a rank interval $[i, j]$, deciding $P \equiv{ }^{s,[i, j]} Q$ is $\Pi_{2}^{P}$-complete in case of $C O$ problems and $\Pi_{3}^{P}$-complete in case of $A S O$ problems.

Proof. [Sketch, a detailed argument is provided in Appendix B.] The membership part essentially follows the same arguments as the proof of Theorem 21, but here the problem of deciding $I \in \pi\left(P_{<i}\right)$ is in co-NP for CO problems and in $\Pi_{2}^{P}$ for ASO problems.

For the hardness part, we reduce the following problem to sel-equivalence of CO problems: Given two propositional theories $S$ and $T$, decide whether they possess the same minimal models. This problem is known to be $\Pi_{2}^{P}$-complete (e.g., Eiter et al., 2007b, Thm. 6.15), and the problem remains hard if $S$ and $T$ are in negation normal form (NNF) given over the same alphabet. We adapt a construction used by Brewka et al., (2011). Given a negation normal form theory $T$, we construct a CO problem $P_{T}$ by setting

$$
\begin{aligned}
& P_{T}^{g}=T\left[\neg u / u^{\prime}\right] \cup\left\{u \leftrightarrow \neg u^{\prime} \mid u \in U\right\}, \text { and } \\
& P_{T}^{s}=\left\{u^{\prime}>u \leftarrow \mid u \in U\right\},
\end{aligned}
$$

where $U$ denotes the set of atoms occurring in $T$, and $T\left[\neg u / u^{\prime}\right]$ stands for the theory resulting from replacing all $\neg u$ by $u^{\prime}$ in $T$ (we note that the ranks of rules in the selector are 1).

The elements in $\pi\left(P_{T}\right)$ are in a one-to-one correspondence to the minimal models of $T$. For theories $S$ and $T$ over $U$ it follows that $S$ and $T$ have the same minimal models if and only if $\pi\left(P_{S}\right)=\pi\left(P_{T}\right)$. Since the problems $P_{S}$ and $P_{T}$ have the same selectors, that latter condition is equivalent to $P_{S} \equiv{ }^{s, \geq 2} P_{T}$ (which can be shown directly or exploiting our characterization of $\equiv^{s, \geq 2}$ ).

Concerning the hardness part for ASO problems, we use the following problem: given two open quantified Boolean formulas (QBFs) $\forall Y \phi(X, Y), \forall Y \psi(X, Y)$, decide whether they possess the same minimal models. This problem is $\Pi_{3}^{P}$-hard (see Lemma 30 in Appendix A). For $\phi(X, Y)$, we construct $P_{\phi}$ as follows:

$$
\begin{aligned}
P_{\phi}^{g}= & \left\{z \vee z^{\prime} \mid z \in X \cup Y\right\} \cup \\
& \left\{\left(y \wedge y^{\prime}\right) \rightarrow w, w \rightarrow y, w \rightarrow y^{\prime} \mid y \in Y\right\} \cup \\
& \left\{\phi\left[\neg z / z^{\prime}\right] \rightarrow w, \neg w \rightarrow w\right\}, \\
P_{\phi}^{s}= & \left\{x^{\prime}>x \leftarrow \mid x \in X\right\},
\end{aligned}
$$


where $\phi\left[\neg z / z^{\prime}\right]$ stands for the formula obtained by replacing all $\neg z$ by $z^{\prime}$ in $\phi(X, Y)$ (again, we stress that the ranks of rules in the selector are 1). The elements in $\pi\left(P_{\phi}\right)$ are in a one-to-one correspondence to the minimal models of $\forall Y \phi(X, Y)$. Now we can reason as above and show that for $\phi$ and $\psi$ over $X \cup Y$, formulas $\forall Y \phi(X, Y)$ and $\forall Y \psi(X, Y)$ have the same minimal models if and only if $P_{\phi} \equiv{ }^{s, \geq 2} P_{\psi}$.

In Theorem 22 the rank interval $[i, j]$ is given as input. When fixing the interval, the hardness results still hold, provided that $i>1$. In fact, the critical condition in Corollary 7 is $\pi\left(P_{<i}\right)=\pi\left(Q_{<i}\right)$; for rank intervals $[1, j]$, the selectors become empty and the condition is reduced to $\mu(P)=\mu(Q)$, which is easier to decide.

Our characterizations imply that all the remaining problems are in co-NP. For strong gen-equivalence, co-NP-hardness follows directly from Theorem 13 and co-NP-completeness of deciding strong equivalence between two propositional theories (for both semantics).

Theorem 23 Given two $C O$ (ASO, respectively) problems $P$ and $Q$, deciding $P \equiv_{g} Q$ is co-NP-complete.

Finally, for the combined case the hardness result follows from Theorem 16 and co-NPcompleteness of deciding strong equivalence of propositional theories.

Theorem 24 Given ranked $C O$ (ASO, respectively) problems $P$ and $Q$, and rank interval $[i, j]$, deciding $P \equiv_{g}^{s,[i, j]} Q$ is co-NP-complete.

By construction, all hardness results hold already for simple optimization problems.

\section{Discussion}

We introduced the formalism of optimization problems, generalizing the principles of ASO programs, in particular, the separation of hard and soft constraints (Brewka et al., 2003). We focused on two important specializations of optimization problems: CO problems and ASO problems. We studied various forms of strong equivalence for these classes of optimization problems, depending on what contexts are considered. Specifically, we considered the following cases: new preference information is added, but the hard constraints remain unchanged (strong sel-equivalence); hard constraints are added but preferences remain unchanged (strong gen-equivalence); both hard constraints and preferences can be added (strong equivalence). To the best of our knowledge, this natural classification of equivalences in preference formalisms has not been studied yet. In certain cases some of these notions coincide (Theorem 18) but this is no longer true when the underlying semantics is changed or ranks in contexts are restricted.

In previous work, the notion of strong equivalence (both hard constraints and preferences can be added) has been studied for logic programs with weak constraints by Eiter et al., (2007a) and logic programs with ordered disjunctions (LPODs) by Faber et al., (2008). While for the former formalism, a separation of strong equivalence into different notions - as suggested here for ASO problems - would be possible (it is instructive to compare Eiter et al., 2007a, Lemma 23, to our results, e.g., Corollary 20), a similar separation for strong equivalence is not straightforward for LPODs. The reason is the syntactic nature of LPOD rules which act like hard constraints and preference rules at the same time. Faber et 
al., (2008) considered strong equivalence with respect to contexts that are logic programs (which is similar to strong gen-equivalence) and the combined case of strong equivalence (called strong equivalence for arbitrary contexts there), but they did not consider any counterpart to the notion of strong sel-equivalence. In fact, it is even unclear whether in every LPOD the generating and selecting modules can be cleanly separated.

In our paper, we established characterizations of all three types of strong equivalence. They exhibit striking similarities. The characterizations of strong sel-equivalence for $\mathrm{CO}$ and ASO problems in Theorem 6 are precisely the same, mirroring the fact that generators are not subject to change. Theorem 13 concerns strong gen-equivalence for $\mathrm{CO}$ and ASO problems. In each case, the characterizations consist of two requirements: the strong equivalence of generators, and the equality of the strict preference relations restricted to the class of models of the generators. The only difference comes from the fact that strong equivalence for classical and the equilibrium-model semantics have different characterizations. Theorem 16 which concerns the combined case of strong equivalence also does not differentiate between $\mathrm{CO}$ and ASO problems other than implicitly (as before, the conditions of strong equivalence are different for the two semantics). Moreover, the characterizations provided by Theorem 16 arise in a rather systematic way from those given in Theorems 6 and 13. This being the case in each of the different semantics we used strongly suggests that there are some abstract principles at play here. We are currently pursuing this direction, conjecturing that this is an inherent feature of preference formalisms with separation of logical and preferential constraints.

Coming back to LPODs, these comments suggest that identifying a "split" representation for that formalism might be of interest. It could lead to alternative characterizations of (combined) strong equivalence derived from the characterizations of the two "one-dimensional" variants.

Next, we note that our results give rise to problem rewriting methods that transform optimization problems into strongly equivalent ones. We provided two simple examples illustrating that application of our results in Example 9 and Corollary 12. Similar examples can be constructed for our results concerning strong gen-equivalence and (combined) strong equivalence. A more systematic study of optimization problem rewriting rules that result in strongly equivalent problems will be a subject of future work.

Finally, we established the complexity of deciding whether optimization problems are strongly equivalent. Notably, in the general case of strong (combined) equivalence the problem is co-NP-complete for both CO and ASO problems. The same holds true for the strong gen-equivalence problem. For the strong sel-equivalence problem, the situation is more complex. When contexts of the form $[1, j]$ or $[1, \infty]$ are considered, the problem of deciding strong sel-equivalence is co-NP-complete for CO problems and $\Pi_{2}^{P}$-complete for ASO problems. If any rank interval is allowed as part of input or if the rank interval is fixed to $[i, j]$, with $i \geq 2$, the problem gets computationally harder: in case of ASO problems, $\Pi_{3}^{P}$-hard; in case of CO problems $\Pi_{2}^{P}$-hard. The difference between CO problems and ASO problems in the case of strong sel-equivalence with respect to contexts consisting of preference rules with ranks in the intervals $[1, j]$ or $[1, \infty]$ comes from the fact that the corresponding concepts of strong sel-equivalence depend, in particular, on whether two theories are equivalent with respect to models (CO problems) and with respect to equilibrium models (ASO problems). These two types of equivalence have different complexities. The jump in the complexity for 
strong sel-equivalence when arbitrary rank intervals $[i, j]$ are allowed or if they are fixed so that $i \geq 2$ comes from the fact that in such cases, the concept depends on properties of the class of outcomes that are optimal with respect to rules of ranks less than $i$, while in other cases it depends on properties of the class of models. Decision problems concerning optimal outcomes (such as: do two theories have the same "optimal" models) are harder than the corresponding versions of the problems for models, explaining the jump. The results for strong sel-equivalence also imply that ranked optimization problems cannot be efficiently simulated by simple optimization problems.

\section{Acknowledgments}

We thank the reviewers for their useful and constructive comments. The first author was supported by Regione Calabria and EU under POR Calabria FESR 2007-2013 within the PIA project of DLVSYSTEM s.r.l., and by MIUR under the PRIN project LoDeN. The second author was supported by the NSF grant IIS-0913459.

\section{Appendix A. Useful Lemmas}

We provide here several lemmas that we use later in the proofs of the results discussed in the main body of the paper.

The first two lemmas are given without proofs, as they are easy consequences of results by Ferraris (2005) and Ferraris and Lifschitz (2005).

Lemma 25 Let $P$ be a theory, $I$ be a classical model of $P$, and let $\Pi[I]=\{a \rightarrow \perp \mid a \in$ $\mathcal{U} \backslash I\} \cup\{\neg a \rightarrow \perp \mid a \in I\}$. Then, $A S(P \cup \Pi[I])=\operatorname{Mod}(P \cup \Pi[I])=\{I\}$.

Lemma 26 Let $P$ be a theory, $I, J$ two of its (classical) models such that $I \neq J$, and let

$$
\begin{aligned}
& \Pi[I, J]=\{a \vee b \mid a \in I, b \in J\} \\
& \cup\{a \vee \neg b \mid a \in I, b \in \mathcal{U} \backslash J\} \\
& \cup \quad\{\neg a \vee b \mid a \in \mathcal{U} \backslash I, b \in J\} \\
& \cup \quad\{\neg a \vee \neg b \mid a \in \mathcal{U} \backslash I, b \in \mathcal{U} \backslash J\} .
\end{aligned}
$$

Then $A S(P \cup \Pi[I, J])=\operatorname{Mod}(P \cup \Pi[I, J]))=\{I, J\}$.

Lemma 27 Let $P$ be an optimization problem, $I \in \pi\left(P_{<j}\right)$, where $j \geq 1$, and let

$$
\left.R_{j}[I]=\{a>\top \stackrel{j}{\leftarrow} \mid a \in I\} \cup\{\neg a>\top \stackrel{j}{\leftarrow} \mid a \in \mathcal{U} \backslash I)\right\} .
$$

Then

1. $I \in \pi\left(P \cup R_{j}[I]\right)$;

2. for every $J$ such that $J \neq I$ and $I \geq^{P_{\leq j}} J, I>^{P \cup R_{j}[I]} J$. 
Proof. When proving (1), to simplify the notation, we write $R$ for $R_{j}[I]$. Since $I \in \pi\left(P_{<j}\right)$, $I \in \mu(P)$. Clearly, $\mu(P \cup R)=\mu(P)$ and so, $I \in \mu(P \cup R)$. To show that $I \in \pi(P \cup R)$, let us consider an arbitrary interpretation $J \in \mu(P \cup R)$ and assume that $J>P \cup R I$. In particular, $J \neq I$ and so, $\operatorname{diff}^{R}(I, J)=j$. If $\operatorname{diff}^{P}(I, J)<j$, then $\operatorname{diff}^{P \cup R}(I, J)<j$.

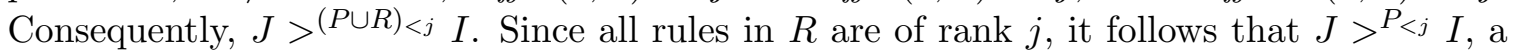
contradiction with the fact that $I \in \pi\left(P_{<j}\right)$. Thus, $\operatorname{diff}^{P}(I, J) \geq j$. Since $\operatorname{diff}^{R}(I, J)=j$, we have $\operatorname{diff}^{P \cup R}(I, J)=j$. Therefore, $J>^{P \cup R} I$ implies $J>^{R} I$, a contradiction again (since, by definition of $R_{j}[I]=R, I \geq^{R} J$ for each interpretation $J$ ). It follows that for every $J \in \mu(P \cup R), J \ngtr P \cup R I$, that is, $I \in \pi(P \cup R)$.

The assertion (2) is evident, since by definition of $R_{j}[I]=R, I>^{R} J$ for each interpretation $J \neq I$.

Lemma 28 Let $P$ be an optimization problem, $I, J$ interpretations such that $I, J \in \pi\left(P_{<j}\right)$, where $j \geq 1$, and let $R_{j}^{\prime}[I, J] \in \mathcal{L}_{\mathcal{U}}^{s, \geq j}$ be the union of the following sets of rules:

$$
\begin{aligned}
& \{a \vee b>\top \stackrel{\leftarrow}{\leftarrow} \mid a, b \in I \cap J\} \\
& \{a \vee \neg b>\top \stackrel{j}{\leftarrow} \mid a \in I, b \in \mathcal{U} \backslash J\} \\
& \{\neg a \vee b>\top \stackrel{j}{\leftarrow} \mid a \in \mathcal{U} \backslash I, b \in J\} \\
& \{\neg a \vee \neg b>\top \stackrel{j}{\leftarrow} \mid a \in \mathcal{U} \backslash I, b \in \mathcal{U} \backslash J\} .
\end{aligned}
$$

Then all of the following hold:

1. for every $r \in R_{j}^{\prime}[I, J], v_{I}(r)=v_{J}(r)=1$;

2. for every interpretation $K \notin\{I, J\}$, there is a rule $r \in R_{j}^{\prime}[I, J]$ such that $v_{K}(r)=2$;

3. $I>^{P} J$ if and only if $J \notin \pi\left(P \cup R_{j}^{\prime}[I, J]\right)$.

Proof. To simplify the notation, we write $R^{\prime}$ for $R_{j}^{\prime}[I, J]$.

The assertion (1) is evident. To prove the assertion (2), we note that the conjunction of all formulas that appear as top options in the preference rules of $R^{\prime}$ is equivalent to

$$
(\bigwedge\{a \mid a \in I\} \wedge \bigwedge\{\neg a \mid a \in \mathcal{U} \backslash I\}) \vee(\bigwedge\{b \mid b \in J\} \wedge \bigwedge\{\neg b \mid b \in \mathcal{U} \backslash J\}) .
$$

That formula has only two models: $I$ and $J$. Thus, for every other interpretation $K$, at least one of the formulas that appears as top options in the preference rules of $R^{\prime}$ is not satisfied by $K$. For the corresponding preference rule $r, v_{K}(r)=2$.

Finally, to prove the assertion (3), let us assume that $I>^{P} J$. Together with (1), it implies that $I>^{P \cup R^{\prime}} J$. Thus, $J \notin \pi\left(P \cup R^{\prime}\right)$. To prove the converse implication, let us assume that $I \ngtr^{P} J$. Together with (1), it implies that $I \ngtr P \cup R^{\prime} J$. Next, we note that if $\operatorname{diff}^{P}(J, K)<j$, then since $J \in \pi\left(P_{<j}\right), K \ngtr P \cup R^{\prime} \quad J$. If $\operatorname{diff}^{P}(J, K) \geq j$, then property (2) proved above implies that $K \ngtr P \cup R^{\prime} J$. Since $K$ is an arbitrary interpretation different from $I$ and $J$, and since $I \not P \cup R^{\prime} J, J \in \pi\left(P \cup R^{\prime}\right)$ follows. 
Next, we note a property that allows us to infer the strong sel-equivalence of two problems treated as CO problems from the strong sel-equivalence of the same problems when treated as ASO problems (and conversely). The property relies on the fact that changing selectors only does not affect the class of outcomes. The proof is simple and we omit it.

Lemma 29 Let $P$ and $Q$ be optimization problems such that $\operatorname{Mod}\left(P^{g}\right)=A S\left(P^{g}\right)$ and $\operatorname{Mod}\left(Q^{g}\right)=A S\left(Q^{g}\right)$, and $[i, j]$ a rank interval. Then, $P \equiv^{s,[i, j]} Q$ when $P$ and $Q$ are viewed as CO problems, if and only if $P \equiv^{s,[i, j]} Q$ when $P$ and $Q$ are viewed as ASO problems.

The final results in this section will be useful for the complexity results.

Lemma 30 Deciding whether open $Q B F s \forall Y \phi(X, Y)$ and $\forall Y \psi(X, Y)$, where $\phi$ and $\psi$ are in negation normal form, have the same minimal models is $\Pi_{3}^{P}$-hard.

Proof. We show the result by a reduction from the $\Pi_{3}^{P}$-hard problem of deciding satisfiability for QBFs of the form $\forall Z \exists X \forall Y \gamma$, where $\gamma$ is in negation normal form. Let $\Phi$ be a QBF of such a form and consider the following formulas, where $Z^{\prime}=\left\{z^{\prime} \mid z \in Z\right\}$, and $u$ and $v$ are fresh atoms:

$$
\begin{aligned}
\phi & =\bigwedge_{z \in Z}\left(z \leftrightarrow \neg z^{\prime}\right) \wedge\left(\left(\bigwedge_{x \in X} x \wedge u\right) \vee \gamma\right) \wedge(v \vee \neg v) \\
\psi & =\bigwedge_{z \in Z}\left(z \leftrightarrow \neg z^{\prime}\right) \wedge\left(\left(\bigwedge_{x \in X} x \wedge v\right) \vee \gamma\right) \wedge(u \vee \neg u) .
\end{aligned}
$$

It is clear that $\phi$ and $\psi$ are in negation normal form. The only difference between $\phi$ compared to $\psi$ is that the latter uses $u$ where the former uses $v$ and vice versa, and the only point of including the conjuncts $v \vee \neg v$ and $u \vee \neg u$ is to have occurrences of $u$ and $v$ in both $\phi$ and $\psi$. We show that $\forall Y \phi(U, Y)$ and $\forall Y \psi(U, Y)$ have the same minimal models (with open variables $U=Z \cup Z^{\prime} \cup X \cup\{u, v\}$ ) if and only if $\Phi$ is true.

We note that when considering models and minimal models of $\forall Y \phi(U, Y)$ and $\forall Y \psi(U, Y)$ we can move the quantifier $\forall Y$ so that it appears directly in front of $\gamma$. It is so because there are no occurrences of atoms from $Y$ in $\phi$ and in $\psi$ outside of $\gamma$.

It is also clear that if $M$ contains neither $u$ nor $v$, then $M$ is a model of $\forall Y \phi(U, Y)$ if and only if $M$ is a model of $\forall Y \psi(U, Y)$. Consequently, for each such $M$ it holds that $M$ is a minimal model of $\forall Y \phi(U, Y)$ if and only if $M$ is a minimal model of $\forall Y \psi(U, Y)$.

Only-if direction: Let us assume $\Phi$ is false. Then, there exists an interpretation $I \subseteq Z$ of atoms in $Z$, such that for every interpretation $J$ of atoms in $X, \forall Y \gamma$ is false. Let us consider $M_{u}=I \cup(Z \backslash I)^{\prime} \cup X \cup\{u\}$. Clearly, $M_{u}$ is a model of $\forall Y \phi$. If $N$ is a model of $\forall Y \phi$ and $N \subseteq M_{u}$, then $I \cup(Z \backslash I)^{\prime} \subseteq N$ because of the conjunct $\bigwedge_{z \in Z}\left(z \leftrightarrow \neg z^{\prime}\right)$. Thus, $N \cap Z=I$. It follows that $\forall Y \gamma$ is false when atoms in $Z$ and $X$ are interpreted by $N$ and, consequently, $X \cup\{u\} \subseteq N$. Thus, $N=M_{u}$, which implies that $M_{u}$ is a minimal model of $\forall Y \phi$. Essentially the same argument shows that $M_{v}=I \cup(Z \backslash I)^{\prime} \cup X \cup\{v\}$ is a minimal model of $\forall Y \psi$. Since $M_{u} \neq M_{v}, \forall Y \phi$ and $\forall Y \psi$ have different minimal models.

If-direction: Let us assume that $\forall Z \exists X \forall Y \gamma$ is true. Let $M$ be a minimal model of $\forall Y \phi$. Clearly, $v \notin M$ (as $M \backslash\{v\}$ is also a model of $\forall Y \phi$ ). Let us assume that $u \in M$. Let us assume in addition that $X \backslash M \neq \emptyset$. These assumptions imply that for the interpretations 
$I=M \cap Z$ of atoms in $Z$ and $J=M \cap X$ of atoms in $X, \forall Y \gamma$ is true. It follows that $M \backslash\{u\}$ is a model of $\forall Y \phi$, a contradiction. Thus, $X \subseteq M$. Let $I=M \cap Z$. Since $\forall Z \exists X \forall Y \gamma$ is true, there is an interpretation $J \subseteq X$ of atoms in $X$ such that $\forall Y \gamma$ is true, with atoms in $Z$ interpreted by $I$ and atoms in $X$ interpreted by $J$. It follows that $I \cup(Z \backslash I)^{\prime} \cup J$ is a model of $\forall Y \phi$. Since $I \cup(Z \backslash I)^{\prime} \cup J \subset M$, this is a contradiction. It follows that $u \notin M$. Consequently, by the comment above, $M$ is a minimal model of $\forall \psi$. The converse holds by the symmetry argument. Thus, the two formulas have the same minimal models.

Lemma 31 Given a ranked preference rule $r$, and an interpretation $I$, calculating $v_{I}(r)$ can be done in polynomial time.

Proof. Initialize a variable $i$ with 1 . Check whether $I \not \models b d(r)$ and if so, halt. Then, check whether $I=h d_{i}(r)$ and if so, halt; otherwise increment $i$ and continue checking; if no more options in the head of $r$ exist, set $i=1$. Each of the checks is a model checking task for a propositional formula and hence in polynomial time. Upon halting, $i$ is equal to $v_{I}(r)$.

Lemma 32 Given an optimization problem $P$ and two interpretations $I, J$, calculating $\operatorname{diff}^{P}(I, J)$ can be done in polynomial time.

Proof. Initialize a variable $x$ with $\infty$, scan the rules in $P^{s}$ and for each ranked preference rule $r \in P^{s}$, determine whether $v_{I}(r) \neq v_{J}(r)$ (in polynomial time due to Lemma 31). If so, set $x$ to $\operatorname{rank}(r)$ if $\operatorname{rank}(r)<x$. After having processed all rules, $x$ is equal to $\operatorname{diff}^{P}(I, J)$.

Lemma 33 Given an optimization problem $P$, and two interpretations $I, J$, deciding whether $I>^{P} J$ holds can be done in polynomial time.

Proof. First, sort the rules in $P^{s}$ by their ranks. Starting from the lowest rank upwards, do the following for each rank $i$ : Check for all rules of rank $i$ whether $v_{I}(r)<v_{J}(r)$ or $v_{I}(r) \leq v_{J}(r)$. If $v_{I}(r)<v_{J}(r)$ holds at least for one rule and $v_{I}(r) \leq v_{J}(r)$ for all other rules of rank $i$, accept. If there are rules $r$ and $r^{\prime}$ of the rank $i$ such that $v_{I}(r)<v_{J}(r)$ and $v_{I}\left(r^{\prime}\right)>v_{J}\left(r^{\prime}\right)$, reject. If all ranks have been processed, reject. By Lemma 31, all steps are doable in polynomial time.

Lemma 34 Given a classical optimization problem $P$ and an interpretation $I$, deciding whether $I \in \pi(P)$ is in co-NP.

Proof. We show that a witness $J$ for the complementary problem (deciding whether $I \notin \pi(P))$ can be verified in polynomial time. If $J=I$, verify in polynomial time that $I$ does not satisfy the propositional theory $P^{g}$, which is well-known to be feasible in polynomial time. Otherwise, verify in polynomial time that $J$ satisfies $P^{g}$ and that $J>^{P} I$ (both in polynomial time, the latter by Lemma 33). 
Lemma 35 Given an answer set optimization problem $P$ and an interpretation $I$, deciding whether $I \in \pi(P)$ is in $\Sigma_{2}^{P}$.

Proof. We show that a witness $J$ for the complementary problem (deciding whether $I \notin \pi(P)$ ) can be verified in polynomial time using an NP oracle. If $J=I$, verify that $I$ does not satisfy $P^{g}$ using the NP oracle. This is possible because answer-set checking is co-NP-complete (Pearce et al., 2009, Thm. 8). Otherwise, verify using the NP oracle that $J$ satisfies the propositional theory $P^{g}$ and that $J>^{P} I$ (in polynomial time by Lemma 33).

\section{Appendix B. Proofs}

Theorem 6 For all ranked optimization problems $P$ and $Q$, and every rank interval $[i, j]$, $P \equiv{ }^{s,[i, j]} Q$ if and only if the following conditions hold:

1. $\pi\left(P_{<i}\right)=\pi\left(Q_{<i}\right)$

2. $>_{\pi\left(P_{<i}\right)}^{P}=>_{\pi\left(Q_{<i}\right)}^{Q}$

3. for every $I, J \in \pi\left(P_{<i}\right)$ such that $i<\operatorname{diff}^{P}(I, J)$ or $i<\operatorname{diff}^{Q}(I, J)$, $\operatorname{diff}^{P}(I, J)=$ $\operatorname{diff}^{Q}(I, J)$ or both $\operatorname{diff}^{P}(I, J)>j$ and $\operatorname{diff}^{Q}(I, J)>j$.

Proof. $\quad(\Leftarrow)$ Let $R \in \mathcal{L}_{\mathcal{U}}^{s,[i, j]}$ and let $I \in \pi(P \cup R)$. By Lemma 3, $I \in \pi\left((P \cup R)_{<i}\right)$. Since $R \in \mathcal{L}_{\mathcal{U}}^{s,[i, j]}, R_{<i}=(\emptyset, \emptyset)$. Thus, $I \in \pi\left(P_{<i}\right)$. By assumption, it follows that $I \in \pi\left(Q_{<i}\right)$. In particular, $I \in \mu\left(Q_{<i}\right)$ and, as $\mu\left(Q_{<i}\right)=\mu(Q), I \in \mu(Q)$. Since $R^{g}=\emptyset$, we have $I \in \mu(Q \cup R)$. To show that $I \in \pi(Q \cup R)$ we have to show that there is no $J \in \mu(Q \cup R)$ such that $J>{ }^{Q \cup R} I$. Let us assume to the contrary that such a $J$ exists. By Lemma 5, there are three possibilities.

First, we assume that $\operatorname{diff}^{Q}(I, J)<\operatorname{diff}^{R}(I, J)$ and $J>^{Q} I$. The latter property implies $\operatorname{diff}^{Q}(I, J) \geq i$ (otherwise, we would have $J>^{Q_{<i}} I$, contrary to $I \in \pi\left(Q_{<i}\right)$ ). In particular, we have $I \approx^{Q_{<i}} J$ and, since $I \in \pi\left(Q_{<i}\right)$, it follows that $J \in \pi\left(Q_{<i}\right)$. By (1), $J \in \pi\left(P_{<i}\right)$. Thus, by (2), $J>^{P} I$. If $\operatorname{diff}^{Q}(I, J) \geq j$ then $\operatorname{diff}^{R}(I, J)>j$ and, as $R \in \mathcal{L}_{\mathcal{U}}^{s,[i, j]}, \operatorname{diff}^{R}(I, J)=\infty$. Since $J>^{P} I, J>^{P \cup R} I$. Otherwise, $i \leq \operatorname{diff}^{Q}(I, J)<j$. If $i<\operatorname{diff}^{Q}(I, J)$ then, by (3), $\operatorname{diff}^{P}(I, J)=\operatorname{diff}^{Q}(I, J)$. If $i=\operatorname{diff}^{Q}(I, J)$, then again by (3), $\operatorname{diff}^{P}(I, J) \leq i$. In either case, $\operatorname{diff}^{P}(I, J)<\operatorname{diff}^{R}(I, J)$. Since $J>^{P} I, J>^{P \cup R} I$.

Next, let us assume that $\operatorname{diff}^{Q}(I, J)>\operatorname{diff}^{R}(I, J)$ and $J>^{R} I$. Since $R \in \mathcal{L}_{\mathcal{U}}^{s,[i, j]}$, it follows that $\operatorname{diff}^{R}(I, J) \geq i$ and so, $\operatorname{diff}^{Q}(I, J)>i$. We recall that $I \in \pi\left(Q_{<i}\right)$. Thus, $J \in \pi\left(Q_{<i}\right)$ and, consequently, $J \in \pi\left(P_{<i}\right)$. If $\operatorname{diff}^{Q}(I, J) \leq j$ then, by (3), $\operatorname{diff}^{P}(I, J)=\operatorname{diff}^{Q}(I, J)$ and so, $\operatorname{diff}^{P}(I, J)>\operatorname{diff}^{R}(I, J)$. If $j<\operatorname{diff}^{Q}(I, J)$ then, also by $(3), j<\operatorname{diff}^{P}(I, J)$. Since $\operatorname{diff}^{Q}(I, J)>\operatorname{diff}^{R}(I, J)$, diff ${ }^{R}(I, J)<\infty$ and, consequently, $\operatorname{diff}^{R}(I, J) \leq j$. Thus, $\operatorname{diff}^{P}(I, J)>\operatorname{diff}^{R}(I, J)$ in this case, too. Since $J>^{R} I, J>^{P \cup R} I$ follows.

Finally, let us assume that $\operatorname{diff}^{Q}(I, J)=\operatorname{diff}^{R}(I, J), J>^{Q} I$ and $J>^{R} I$. Since $R \in \mathcal{L}_{\mathcal{U}}^{s,[i, j]}, \operatorname{diff}^{R}(I, J) \geq i$. Thus, $\operatorname{diff}^{Q}(I, J) \geq i$ and, since $I \in \pi\left(Q_{<i}\right), J \in \pi\left(Q_{<i}\right)$. By (1) we have $J \in \pi\left(P_{<i}\right)$ and, by $(2), J>^{P} I$. Consequently, $J>^{P \cup R} I$.

In all cases we obtained $J>^{P \cup R} I$, contrary to $I \in \pi(P \cup R)$, a contradiction. 
$(\Rightarrow)$ Let us assume that $\pi\left(P_{<i}\right) \neq \pi\left(Q_{<i}\right)$. Without loss of generality, we can assume that there is $I \in \pi\left(P_{<i}\right) \backslash \pi\left(Q_{<i}\right)$ and define $R=\left(\emptyset, R_{i}[I]\right) \in \mathcal{L}_{\mathcal{U}}^{s,=i}$, where $R_{i}[I]$ is as in Lemma 27. By that lemma, $I \in \pi(P \cup R)$. On the other hand, since $I \notin \pi\left(Q_{<i}\right)$ and $R \in \mathcal{L}_{\mathcal{U}}^{s,=i}, I \notin \pi\left((Q \cup R)_{<i}\right)$. By Lemma $3, I \notin \pi(Q \cup R)$. Thus, $P \not \equiv^{s,=i} Q$, contrary to the assumption.

It follows that $\pi\left(P_{<i}\right)=\pi\left(Q_{<i}\right)$, that is, that condition (1) holds. To prove condition (2), let us consider interpretations $I, J \in \pi\left(P_{<i}\right)$ such that $I>^{P} J$. Let $R_{i}^{\prime}[I, J]$ be the selector defined in Lemma 28. Since $I>^{P} J$, Lemma 28(3) implies that $J \notin \pi\left(P \cup R_{i}^{\prime}[I, J]\right)$. Consequently, $J \notin \pi\left(Q \cup R_{i}^{\prime}[I, J]\right)$. By Lemma 28(3) again, it follows that $I>{ }^{Q} J$. By symmetry, $I>^{Q} J$ implies $I>^{P} J$ and so, condition (2) holds.

To prove condition (3), let us assume that there are interpretations $I$ and $J$ that satisfy the assumptions but violate the corresponding conclusion. In what follows, we write $p$ for $\operatorname{diff}^{P}(I, J)$ and $q$ for $\operatorname{diff}^{Q}(I, J)$. Thus, we have $p>i$ or $q>i, p \neq q$, and $p \leq j$ or $q \leq j$. Without loss of generality, we can assume that $p<q$. It follows that $p$ is finite and, consequently, that $\operatorname{diff}^{P}(I, J)<\infty$ and $I \neq J$. Moreover, $i<q$ and $p \leq j$.

Let us assume first that $p<i$. We take the problem $R=\left(\emptyset, R_{i}[J]\right)$, where $R_{i}[J]$ is as specified in Lemma 27 and define $P^{\prime}=P \cup R$ and $Q^{\prime}=Q \cup R$. Since $I, J \in \pi\left(P_{<i}\right)$ we also have $I, J \in \pi\left(Q_{<i}\right)$. By our assumptions, $q>i$. Thus, $J \approx^{Q_{\leq i}} I$ and, in particular, $J \geq^{Q_{\leq i}} I$. We recall that $I \neq J$. Consequently, by the assertion (2) of Lemma 27 we have that $J>^{Q^{\prime}} I$. Since all rules in $R$ have ranks $i$, we have $I, J \in \pi\left(P_{<i}^{\prime}\right)$ and $\operatorname{diff}^{P^{\prime}}(I, J)=$ $\operatorname{diff}^{P}(I, J)<i$. It follows that $J \ngtr^{P^{\prime}} I$ (otherwise, by $\operatorname{diff}^{P^{\prime}}(I, J)<i$ we would have $\left.J>P_{<i}^{\prime} I\right)$. Let us define $R^{\prime}=\left(\emptyset, R_{i}^{\prime}[I, J]\right)$, where $R_{i}^{\prime}[I, J]$ is as specified in Lemma 28. Since $J \ngtr P^{\prime} I$, by the assertion (3) of that lemma, $I \in \pi\left(P^{\prime} \cup R^{\prime}\right)$. We have $P^{\prime} \cup R^{\prime}=P \cup\left(R \cup R^{\prime}\right)$. Thus, $I \in \pi\left(P \cup\left(R \cup R^{\prime}\right)\right)$ and, by $P \equiv^{s,[i, j]} Q, I \in \pi\left(Q \cup\left(R \cup R^{\prime}\right)\right)=\pi\left(Q^{\prime} \cup R^{\prime}\right)$. By the assertion (3) of Lemma 28, $J \ngtr^{Q^{\prime}} I$, a contradiction.

Next, let $p=i$. Clearly, $I \ngtr^{P} J$ or $J \ngtr^{P} I$. Without loss of generality, let us assume that $J \ngtr^{P} I$. Let $R^{\prime}=\left(\emptyset, R_{i}[I, J]\right)$, and let us define $P^{\prime}=P \cup R^{\prime}$ and $Q^{\prime}=Q \cup R^{\prime}$. Since all rules in $R^{\prime}$ have ranks $i, I, J \in \pi\left(P_{<i}\right)$ implies $I, J \in \pi\left(P_{<i}^{\prime}\right)$. Moreover, from $J \ngtr^{P} I$ it follows by Lemma 28(1) that $J \ngtr^{P^{\prime}} I$. Let $R=\left(\emptyset, R_{i}[J]\right)$. All rules in $R$ have rank $i$ and $\operatorname{diff}^{P^{\prime}}(I, J)=\operatorname{diff}^{P}(I, J)=i$. Thus, it follows that $J \ngtr P^{P^{\prime} \cup R} I$. Moreover, for every $K \notin\{I, J\}$, if $\operatorname{diff}^{P^{\prime}}(K, I)<i$, then $K \ngtr^{P^{\prime} \cup R} I$ follows from $I \in \pi\left(P_{<i}^{\prime}\right)$. If $\operatorname{diff}^{P^{\prime}}(K, I) \geq i$, then $K \ngtr P^{\prime} \cup R I$ follows from Lemma 28(2). Thus, $I \in \pi\left(P^{\prime} \cup R\right)$. On the other hand, we recall that $\operatorname{diff}^{Q}(I, J)=q>i$. Thus, $\operatorname{diff}^{Q^{\prime}}(I, J)>i$, too (Lemma 28(1)). It follows that $J \geq^{Q_{\leq i}^{\prime}} I$. Consequently, by Lemma $27(2)$, we have $J>^{Q^{\prime} \cup R} I$. Thus, $I \notin \pi\left(Q^{\prime} \cup R\right)$, a contradiction.

It follows that $p>i$. To complete the proof of (3), we recall that $p \leq j$. Clearly, $I \ngtr^{P} J$ or $J \ngtr^{P} I$. Without loss of generality, let us assume that $J \ngtr^{P} I$. Let $R^{\prime}=\left(\emptyset, R_{i}[I, J]\right)$, and let us define $P^{\prime}=P \cup R^{\prime}$ and $Q^{\prime}=Q \cup R^{\prime}$. Let us assume that for some interpretation $K \notin$ $\{I, J\}, K>^{P^{\prime}} I$. By Lemma 28(2), it follows that $\operatorname{diff}^{P^{\prime}}(I, K)<i$. Thus, $\operatorname{diff}^{P}(I, K)<i$, a contradiction with $I \in \pi\left(P_{<i}\right)$. Thus, for every interpretation $K \notin\{I, J\}, K \ngtr^{P^{\prime}} I$ and, by the same argument, $K \ngtr^{P^{\prime}} J$. Consequently, for every interpretation $K \notin\{I, J\}$, $K \ngtr P^{\prime}<p I$ and $K \ngtr^{P_{<p}^{\prime}} J$. In addition, since $I, J \in \pi\left(P_{<i}\right)$, by Lemma 28(1) we obtain that neither $I>^{P_{<p}^{\prime}} J$ nor $J>^{P_{<p}^{\prime}} I$. Thus, $I, J \in \pi\left(P_{<p}^{\prime}\right)$. In addition, by Lemma 28(1), $\operatorname{diff}^{P^{\prime}}(I, J)=\operatorname{diff}^{P}(I, J)=p$ and, since $J \ngtr^{P} I, J \ngtr^{P^{\prime}} I$. 
Let $R=\left(\emptyset, R_{p}[J]\right)$. As (i) $\operatorname{diff}^{P^{\prime}}(I, J)=p$, (ii) $J \ngtr^{P^{\prime}} I$, and (iii) all rules in $R$ have rank $p$, it follows that $J \ngtr^{P^{\prime} \cup R} I$. Moreover, for every $K \notin\{I, J\}$, if $\operatorname{diff}^{P^{\prime}}(K, I)<i$, then $K \ngtr P^{\prime} \cup R I$ follows from $I \in \pi\left(P_{<i}^{\prime}\right)$. If $\operatorname{diff}^{P^{\prime}}(K, I) \geq i$, then $K \ngtr^{P^{\prime} \cup R} I$ follows from Lemma 28(2) (and the definition of $\left.P^{\prime}\right)$. Thus, $I \in \pi\left(P^{\prime} \cup R\right)$. On the other hand, we recall that $\operatorname{diff}^{Q}(I, J)=q>p$. Thus, $\operatorname{diff}^{Q^{\prime}}(I, J)>p$, too (Lemma 28(1)). It follows that $J \geq^{Q_{\leq p}^{\prime}} I$. Consequently, by Lemma $27(2)$, we have $J>^{Q^{\prime} \cup R} I$. Thus, $I \notin \pi\left(Q^{\prime} \cup R\right)$, a contradiction (we recall that $P \equiv^{s,[i, j]} Q, P^{\prime}=P \cup\left(R \cup R^{\prime}\right), Q^{\prime}=Q \cup\left(R \cup R^{\prime}\right)$, and, as $\left.p \leq j, R \cup R^{\prime} \in \mathcal{L}_{\mathcal{U}}^{s,[i, j]}\right)$.

Theorem 13 For all $C O$ (ASO, respectively) problems $P$ and $Q, P \equiv_{g} Q$ if and only if $P^{g}$ and $Q^{g}$ are strongly equivalent (that is, $\operatorname{Mod}\left(P^{g}\right)=\operatorname{Mod}\left(Q^{g}\right)$ for $C O$ problems, and $\operatorname{Mod}_{H T}\left(P^{g}\right)=\operatorname{Mod}_{H T}\left(Q^{g}\right)$ for ASO problems) and $>_{\operatorname{Mod}\left(P^{g}\right)}^{P}=>_{M o d}^{Q}\left(Q^{g}\right)$.

Proof. $\quad(\Leftarrow)$ The first assumption implies strong equivalence of the generators $P^{g}$ and $Q^{g}$ relative to the corresponding semantics (we recall that in the case of classical semantics, strong and standard equivalence coincide). It follows that for every problem $R \in \mathcal{L}_{\mathcal{U}}^{g}$, $\mu(P \cup R)=\mu(Q \cup R)$. Moreover, for each semantics, $\mu(P \cup R) \subseteq \operatorname{Mod}\left(P^{g} \cup R^{g}\right) \subseteq \operatorname{Mod}\left(P^{g}\right)$ and, similarly, $\mu(Q \cup R) \subseteq \operatorname{Mod}\left(Q^{g} \cup R^{g}\right) \subseteq \operatorname{Mod}\left(Q^{g}\right)$. Since $>_{\operatorname{Mod}\left(P^{g}\right)}^{P}=>_{\operatorname{Mod}\left(Q^{g}\right)}^{Q^{2}}$, and $R \in \mathcal{L}_{\mathcal{U}}^{g}$ does not change preferences, we have $>_{\mu\left(P^{g} \cup R^{g}\right)}^{P \cup R}=>_{\mu\left(Q^{g} \cup R^{g}\right)}^{Q \cup R}$. By Lemma 2, $\pi(P \cup R)=\pi(Q \cup R)$.

$(\Rightarrow)$ Let us assume that $P^{g}$ and $Q^{g}$ are not strongly equivalent. Then, there is a problem $R \in \mathcal{L}_{\mathcal{U}}^{g}$ such that $\mu(P \cup R) \neq \mu(Q \cup R)$. Without loss of generality, we can assume that for some interpretation $I, I \in \mu(P \cup R) \backslash \mu(Q \cup R)$. Let us define a problem $T \in \mathcal{L}_{\mathcal{U}}^{g}$ by setting $T=(\Pi[I], \emptyset)$, where $\Pi[I]$ is as defined in Lemma 25. By that lemma, $\mu(P \cup R \cup T)=\{I\}$ and $\mu(Q \cup R \cup T)=\emptyset$. The former property implies that $I$ is necessarily preferred, that is $I \in \pi(P \cup R \cup T)$, and the latter one implies that $\pi(Q \cup R \cup T)=\emptyset$. This is a contradiction with the assumption that $P \equiv_{g} Q$. Thus, $P^{g}$ and $Q^{g}$ are strongly equivalent, that is, $\operatorname{Mod}\left(P^{g}\right)=$ $\operatorname{Mod}\left(Q^{g}\right)$, in the case $P$ and $Q$ are CO problems, and $\operatorname{Mod}_{H T}\left(P^{g}\right)=\operatorname{Mod}_{H T}\left(Q^{g}\right)$, in the case $P$ and $Q$ are ASO problems.

Since $\operatorname{Mod}_{H T}\left(P^{g}\right)=\operatorname{Mod}_{H T}\left(Q^{g}\right)$ implies $\operatorname{Mod}\left(P^{g}\right)=\operatorname{Mod}\left(Q^{g}\right)$, the latter identity holds in each of the two cases. Because of the equality, we will write $M$ for both $\operatorname{Mod}\left(P^{g}\right)$ and $\operatorname{Mod}\left(Q^{g}\right)$. It remains to show that $>_{M}^{P}=>_{M}^{Q}$. Towards a contradiction, let us assume that there are $I, J \in M$ that are in exactly one of these two relations; without loss of generality we will assume that $I>^{P} J$ and $I \ngtr^{Q} J$. The former identity implies, in particular, that $I \neq J$. Let $T=(\Pi[I, J], \emptyset)$, where $\Pi[I, J]$ is a theory defined in Lemma 26. By that lemma, $\mu(P \cup T)=\mu(Q \cup T)=\{I, J\}$. Clearly, $J \notin \pi(P \cup T)$ and $J \in \pi(Q \cup T)$, contrary to our assumption that $P \equiv{ }_{g} Q$.

Theorem 16 For all ranked $C O$ (ASO, respectively) problems $P$ and $Q$, and every rank interval $[i, j], P \equiv_{g}^{s,[i, j]} Q$ if and only if the following conditions hold:

1. $P^{g}$ and $Q^{g}$ are strongly equivalent (that is, $\operatorname{Mod}\left(P^{g}\right)=\operatorname{Mod}\left(Q^{g}\right)$ for $C O$ problems, and $\operatorname{Mod}_{H T}\left(P^{g}\right)=\operatorname{Mod}_{H T}\left(Q^{g}\right)$ for ASO problems)

2. $>_{\operatorname{Mod}\left(P^{g}\right)}^{P}=>_{\operatorname{Mod}\left(Q^{g}\right)}^{Q}$ 
3. for every $I, J \in \operatorname{Mod}\left(P^{g}\right)$ such that $i<\operatorname{diff}^{P}(I, J)$ or $i<\operatorname{diff}^{Q}(I, J)$, $\operatorname{diff}^{P}(I, J)=$ $\operatorname{diff}^{Q}(I, J)$ or both $\operatorname{diff}^{P}(I, J)>j$ and $\operatorname{diff}^{Q}(I, J)>j$

4. $>_{\operatorname{Mod}\left(P^{g}\right)}^{P_{<i}}=>_{\operatorname{Mod}\left(Q^{g}\right)}^{Q_{<i}}$.

Proof. $(\Leftarrow)$ By Proposition 4, it suffices to prove that for every $R \in \mathcal{L}_{\mathcal{U}}^{g}, P \cup R \equiv^{s,[i, j]} Q \cup R$. By (1), we have that $\mu(P \cup R)=\mu(Q \cup R)$ (we recall that $\mu(P)$ denotes the set of outcomes of an optimization problem $P ; \mu(P)=\operatorname{Mod}\left(P^{g}\right)$ in the case of CO problems, and $\mu(P)=A S\left(P^{g}\right)$ in the case of ASO problems). Moreover, for each type of problems, we also have $\mu(P \cup R) \subseteq \operatorname{Mod}\left(P^{g} \cup R^{g}\right) \subseteq \operatorname{Mod}\left(P^{g}\right)$ and, similarly, $\mu(Q \cup R) \subseteq \operatorname{Mod}\left(Q^{g} \cup R^{g}\right) \subseteq$ $\operatorname{Mod}\left(Q^{g}\right)$. Since all rules in $R$ have rank at least $i$, by condition (4) it follows that $>_{\mu(P \cup R)}^{(P \cup R)_{<i}}=>_{\mu(Q \cup R)}^{(Q \cup R)_{<i}}$. By Lemma 2, we have $\pi\left((P \cup R)_{<i}\right)=\pi\left((Q \cup R)_{<i}\right)$ and so, condition (1) of Theorem 6 holds for $P \cup R$ and $Q \cup R$. Since $\pi\left((P \cup R)_{<i}\right) \subseteq \mu(P \cup R) \subseteq \operatorname{Mod}\left(P^{g}\right)$, and since the corresponding inclusions hold for $Q$, too, conditions $(2)-(3)$ of this theorem for $P$ and $Q$ imply conditions (2)-(3) from Theorem 6 for $P \cup R$ and $Q \cup R$. Thus, by Theorem 6, $P \cup R \equiv^{s,[i, j]} Q \cup R$.

$(\Rightarrow)$ Let us assume that $P \equiv_{g}^{s,[i, j]} Q$. Then, $P \equiv_{g} Q$ follows and, by Theorem 13, implies the appropriate version of condition (1). Since $\operatorname{Mod}_{H T}\left(P^{g}\right)=\operatorname{Mod}_{H T}\left(Q^{g}\right) \operatorname{implies} \operatorname{Mod}\left(P^{g}\right)=$ $\operatorname{Mod}\left(Q^{g}\right)$, for each of the two versions of the assertion we have $\operatorname{Mod}\left(P^{g}\right)=\operatorname{Mod}\left(Q^{g}\right)$. From now on in the proof, we write $M$ for $\operatorname{Mod}\left(P^{g}\right)$ and, because of the equality, also for $\operatorname{Mod}\left(Q^{g}\right)$.

Next, for interpretations $I, J \in M$ such that $I \neq J$, we define $R=(\Pi[I, J], \emptyset)$, where $\Pi[I, J]$ is as in Lemma 26. Let us define $P_{1}=P \cup R$ and $Q_{1}=Q \cup R$. We have $P_{1} \equiv^{s,[i, j]} Q_{1}$. Moreover, by Lemma 26, we also have that $\mu\left(P_{1}\right)=\mu\left(Q_{1}\right)=\{I, J\}$.

To prove condition (4), let us assume that $I>^{P_{<i}} J$. It follows that $I>^{P_{1}} J$ (we recall that $R$ contains no preference rules). Since $\mu\left(P_{1}\right)=\{I, J\}, J \notin \pi\left(P_{1}\right)$ and $I \in \pi\left(P_{1}\right)$. By the assumption, $J \notin \pi\left(Q_{1}\right)$. Since $\mu\left(Q_{1}\right)=\{I, J\}$, we have that $I>^{Q_{1}} J$. In particular, $I \in \pi\left(Q_{1}\right)$. If $\operatorname{diff}^{Q}(I, J)<i$ then, since $R$ has no preference rules, $I>^{Q_{<i}} J$. Thus, let us assume that $\operatorname{diff}^{Q}(I, J) \geq i$ and let us define $R^{\prime}=\left(\emptyset, R_{i}[J]\right)$, where $R_{i}[J]$ is as in Lemma 27. Since (i) $I>^{P_{<i}} J$, (ii) $R$ has no preference rules, and (iii) all preference rules in $R^{\prime}$ have rank $i$, it follows that $I>P_{1} \cup R^{\prime} J$. The generator module in $R^{\prime}$ is empty. It follows that $\mu\left(P_{1} \cup R^{\prime}\right)=\mu\left(Q_{1} \cup R^{\prime}\right)=\{I, J\}$. Thus, $J \notin \pi\left(P_{1} \cup R^{\prime}\right)$ and, consequently, $J \notin \pi\left(Q_{1} \cup R^{\prime}\right)$. Since $\operatorname{diff}^{Q}(I, J) \geq i$, $\operatorname{diff}^{Q_{1}}(I, J) \geq i$. Moreover, $I \in \pi\left(Q_{1}\right)$ and so, also $I \in \pi\left(\left(Q_{1}\right)_{<i}\right)$. Thus, $J \in \pi\left(\left(Q_{1}\right)_{<i}\right)$. By Lemma $27, J \in \pi\left(Q_{1} \cup R^{\prime}\right)$, a contradiction. The argument shows that $I>^{P_{<i}} J$ implies $I>^{Q_{<i}} J$. The converse implication follows by symmetry and so, condition (4) holds.

To prove condition (2), let us assume that $I>^{P} J$. If $\operatorname{diff}^{P}(I, J)<i$, then $I>^{P<i} J$ and, by (4), $I>^{Q_{<i}} J$. Thus, $I>^{Q} J$. Let us assume then that $\operatorname{diff}^{P}(I, J) \geq i$. Since $\mu\left(P_{1}\right)=\{I, J\}$ and since $I>^{P} J$ implies $I>^{P_{1}} J, J \notin \pi\left(P_{1}\right)$. Thus, $J \notin \pi\left(Q_{1}\right)$. Since $\mu\left(Q_{1}\right)=\{I, J\}, I>^{Q_{1}} J$ and so, $I>^{Q} J$.

To prove condition (3), without loss of generality we assume that $i<\operatorname{diff}^{P}(I, J)$. Thus, we also have $i<\operatorname{diff}^{P_{1}}(I, J)$ and that $I, J \in \pi\left(\left(P_{1}\right)_{<i}\right)$, the latter follows from the properties that $\mu\left(P_{1}\right)=\{I, J\}$ and that $\operatorname{diff}^{P_{1}}(I, J)=\operatorname{diff}^{P}(I, J)>i$. Since $P_{1} \equiv^{s,[i, j]} Q_{1}$, condition (3) of Theorem 6 holds for $P_{1}, Q_{1}, I$ and $J$, that is, $\operatorname{diff}^{P_{1}}(I, J)=\operatorname{diff}^{Q_{1}}(I, J)$ or both $\operatorname{diff}^{P_{1}}(I, J)>j$ and $\operatorname{diff}^{Q_{1}}(I, J)>j$. Consequently, $\operatorname{diff}^{P}(I, J)=\operatorname{diff}^{Q}(I, J)$ or both 
$\operatorname{diff}^{P}(I, J)>j$ and $\operatorname{diff}^{Q}(I, J)>j$, that is, condition (3) holds.

Theorem 21 Given optimization problems $P$ and $Q$, deciding $P \equiv{ }^{s} Q$ is co-NP-complete in case of $C O$ problems and $\Pi_{2}^{P}$-complete in case of $A S O$ problems.

Proof. For membership, we consider the complementary problem. By Corollary 8, it can be specified as the problem to decide whether at least one of the conditions (1) - (3) below holds:

1. $\mu(P) \neq \mu(Q)$, or equivalently, for some $I$, exactly one of the identities $I \in \mu(P)$ and $I \in \mu(Q)$ holds;

2. for some $I, J \in \mu(P) \operatorname{diff}^{P}(I, J) \neq \operatorname{diff}^{Q}(I, J)$;

3. $>_{\mu(P)}^{P} \neq>_{\mu(Q)}^{Q}$, or equivalently, for some $I, J$, we have $I, J \in \mu(P) \cap \mu(Q)$ and exactly one of the properties $I>^{P} J$ and $I>^{Q} J$ holds.

We show that the problem is in NP for CO problems and in $\Sigma_{2}^{P}$ for ASO problems. We say that a pair of interpretations $I, J$ is a witness for an instance of that problem to be a YES instance if it demonstrates one of the conditions (1) - (3) to hold. It is easy to see that if a witness exists, then there is a witness $I, J$ such that $I$ and $J$ consist only of atoms that occur in $P$ and $Q$. Once such a witness is guessed, condition (1) can be verified in polynomial time for $\mathrm{CO}$ problems (this is a model-checking problem for an interpretation and a theory) and, for ASO problems, in polynomial time with the assist of two calls to an NP oracle, since model checking for the equilibrium-model semantics is co-NP-complete (Theorem 8, Pearce et al. (2009)). Since condition (2) can be verified in polynomial time by Lemma 32, and condition (3) in polynomial time for CO problems and in polynomial time with the assist of four calls to an NP oracle for ASO problems (Lemma 33 and the result by Pearce et al., (2009) mentioned above), the membership part of the assertion follows.

For hardness, we observe that in case of problems with empty selectors, $\equiv^{s}$ coincides with equivalence of propositional theories in case of $\mathrm{CO}$ problems, and with equivalence of equilibrium theories in case of ASO problems. The former is well known to be co-NP-hard, the latter is $\Pi_{2}^{P}$-hard (Theorem 11, Pearce et al. (2009)).

Theorem 22 Given optimization problems $P$ and $Q$ and rank interval $[i, j]$, deciding $P \equiv{ }^{s,[i, j]} Q$ is $\Pi_{2}^{P}$-complete in case of $C O$ problems and $\Pi_{3}^{P}$-complete in case of $A S O$ problems.

Proof. To prove the membership part, we consider the complementary problem. By Theorem 6, that problem consists of deciding whether it is the case that at least one of the following conditions holds:

1. $\pi\left(P_{<i}\right) \neq \pi\left(Q_{<i}\right)$, or equivalently, for some interpretation $I$, exactly one of the properties $I \in \pi\left(P_{<i}\right)$ and $I \in \pi\left(Q_{<i}\right)$ holds;

2. $>_{\pi\left(P_{<i}\right)}^{P} \neq>_{\pi\left(Q_{<i}\right)}^{Q}$, or equivalently, for some interpretations $I, J \in \pi\left(P_{<i}\right) \cap \pi\left(Q_{<i}\right)$ exactly one of the properties $I>^{P} J$ or $I>^{Q} J$ holds;

3. for some $I, J \in \pi\left(P_{<i}\right) \cap \pi\left(Q_{<i}\right)$ such that $i<\operatorname{diff}^{P}(I, J)$ or $i<\operatorname{diff}^{Q}(I, J)$, $\operatorname{diff}^{P}(I, J) \neq \operatorname{diff}^{Q}(I, J)$ and it holds that $\operatorname{diff}^{P}(I, J) \leq j$ or $\operatorname{diff}^{Q}(I, J) \leq j$. 
We call each pair of interpretations $I, J$ that demonstrates that one of the conditions above holds a witness. It is easy to see that if there is witness, there is also one that consists only of atoms that occur in $P$ and $Q$. We show that the complementary problem is in $\Sigma_{2}^{P}$ for $\mathrm{CO}$ problems and in $\Sigma_{3}^{P}$ for ASO problems by showing that a witness once "guessed" can be verified in polynomial time, using an NP oracle when dealing with a CO problem and a $\Sigma_{2}^{P}$ oracle when dealing with an ASO problem.

Consider a witness $I, J$ of two interpretations. One can test whether $I, J$ verifies condition (1) (only $I$ matters here) in polynomial time with two calls to an NP oracle for CO problems (Lemma 34), and with two calls to a $\Sigma_{2}^{P}$ oracle for ASO problems (Lemma 35). To verify condition (2), we need four calls to the respective oracles to test that $I, J \in \pi\left(P_{<i}\right) \cap \pi\left(Q_{<i}\right)$ and polynomial-time computation to test that exactly one of the properties $I>^{P} J$ or $I>^{Q} J$ holds (Lemma 33). Similarly, for condition (3), we use four calls to the respective oracles to test that $I, J \in \pi\left(P_{<i}\right) \cap \pi\left(Q_{<i}\right)$ and polynomial-time computation to test the condition involving $\operatorname{diff}^{P}(I, J)$ and $\operatorname{diff}^{Q}(I, J)$ (Lemma 32).

For the hardness part, we start with the case of CO problems. Therefore, we reduce the following problem to strong sel-equivalence: given two propositional theories $S$ and $T$, decide whether they possess the same minimal models. This problem is known to be $\Pi_{2}^{P}$-complete (for instance, equivalence for positive disjunctive programs is known to $\Pi_{2}^{P}$-complete, see e.g., Eiter et al., 2007b, Thm. 6.15, which means testing whether two propositional formulas of a particular class have the same minimal models). The problem remains hard if $S$ and $T$ are in negation normal form over the same alphabet. Given a negation normal form theory $T$ we construct a $\mathrm{CO}$ problem $P_{T}$ such that the elements in $\pi\left(P_{T}\right)$ are in a one-to-one correspondence to the minimal models of $T$. We adapt a construction used in (Brewka et al., 2011). Specifically, we set

$$
P_{T}^{g}=T\left[\neg u / u^{\prime}\right] \cup\left\{u \leftrightarrow \neg u^{\prime} \mid u \in U\right\},
$$

where $U$ is the collection of atoms occurring in $T$, and $T\left[\neg u / u^{\prime}\right]$ stands for replacing all $\neg u$ by $u^{\prime}$ in $T$, and

$$
P_{T}^{s}=\left\{u^{\prime}>u \leftarrow \mid u \in U\right\} .
$$

Our first observation is that each outcome of $P_{T}$ must be of the form $I \cup\left\{y^{\prime} \mid y \in U \backslash I\right\}$ where $I \subseteq U$. For each interpretation $I \subseteq U$ we write $I^{+}=I \cup\left\{y^{\prime} \mid y \in U \backslash I\right\}$. It is clear that $I \models \neg u$ if and only if $I^{+} \models u^{\prime}$, and hence also $I \models T$ if and only if $I^{+} \models T\left[\neg u / u^{\prime}\right]$. Hence there is a one-to-one mapping between models $M$ of $T$ and outcomes $M^{+}$of $P_{T}$.

Now let us assume that $M^{+} \in \pi\left(P_{T}\right)$. Then $M \models T$, and for any $N \subset M$ we we have $N \not \models T$. Indeed if $N \models T$, then $N^{+} \models P_{T}^{g}$ and for rules $r \in P_{T}^{s}$ of the form $u^{\prime}>u$, where $u \in M \backslash N$, we obtain $v_{N^{+}}(r)=1<2=v_{M^{+}}(r)$ and for all other rules $r^{\prime}$ in $P_{T}^{s}$ we have $v_{N^{+}}\left(r^{\prime}\right)=2=v_{M^{+}}\left(r^{\prime}\right)$. That implies $N^{+}>^{P_{T}} M^{+}$, contradicting $M^{+} \in \pi\left(P_{T}\right)$. Thus, $M$ is a minimal model of $T$.

Conversely, let us assume that $M$ is a minimal model of $T$. Then $M^{+} \in \mu\left(P_{T}\right)$ and for all $N^{+} \in \mu\left(P_{T}\right)$ we have that $N^{+}>^{P_{T}} M^{+}$does not hold, implying $M^{+} \in \pi\left(P_{T}\right)$. Indeed, if $N^{+}>^{P_{T}} M^{+}$, then $v_{N^{+}}(r)<v_{M^{+}}(r)$ for at least one $r \in P_{T}^{s}$ and $v_{N^{+}}\left(r^{\prime}\right) \leq v_{M^{+}}\left(r^{\prime}\right)$ for all $r^{\prime} \in P_{T}^{s}$. The latter implies $N \subseteq M$ and the former shows that $N \neq M$. Since $N \models T$ it contradicts the assumption that $M$ is a minimal model of $T$. It follows that $M^{+} \in \pi\left(P_{T}\right)$.

We thus have that $M^{+} \in \pi\left(P_{T}\right)$ if and only if $M$ is a minimal model of $T$. Moreover, for $S$ and $T$ over $U$ it follows that $S$ and $T$ have the same minimal models if and only 
if $P_{S} \equiv{ }^{s, \geq 2} P_{T}$. Indeed, for any $R \in \mathcal{L}_{\mathcal{U}}^{s, \geq 2}$ it is easy to verify that $\pi\left(P_{S}\right)=\pi\left(P_{S} \cup R\right)$ and $\pi\left(P_{T}\right)=\pi\left(P_{T} \cup R\right)$. That observation follows from the fact that there are no distinct $I, J \in \mu\left(P_{S}\right)$ such that $I \approx^{P_{S}} J$ and no distinct $I, J \in \mu\left(P_{T}\right)$ such that $I \approx^{P_{T}} J$. Thus, rules in $R$ which, being of weaker rank, can only break ties, do not affect the sets of optimal outcomes.

Concerning hardness for ASO problems, we can use a similar idea. However, we shall use the following problem: given two open QBFs $\forall Y \phi(X, Y), \forall Y \psi(X, Y)$, decide whether these two QBFs possess the same minimal models. By Lemma 30, this problem is $\Pi_{3}^{P}$-hard and, moreover, we can assume that $\phi$ and $\psi$ are in negation normal form. The reduction then combines the idea from above with the reduction for general ASP consistency (Eiter \& Gottlob, 1995). More precisely, for each $z \in X \cup Y$ we introduce a new variable $z^{\prime}$, and then we construct $P_{\phi}$ for a given $\phi(X, Y)$ as follows:

$$
\begin{aligned}
P_{\phi}^{g}= & \left\{z \vee z^{\prime} \mid z \in X \cup Y\right\} \cup \\
& \left\{\left(y \wedge y^{\prime}\right) \rightarrow w, w \rightarrow y, w \rightarrow y^{\prime} \mid y \in Y\right\} \cup \\
& \left\{\phi\left[\neg z / z^{\prime}\right] \rightarrow w, \neg w \rightarrow w\right\},
\end{aligned}
$$

where $\phi\left[\neg z / z^{\prime}\right]$ stands for replacing all $\neg z$ by $z^{\prime}$, and $w$ is a globally new atom. For the selector we set

$$
P_{\phi}^{s}=\left\{x^{\prime}>x \leftarrow \mid x \in X\right\} .
$$

Any equilibrium model $M$ of $P_{\phi}^{g}$ must contain $w$ (otherwise $\neg w \rightarrow w$ would be unsatisfied), and it must also contain all of $\left\{y, y^{\prime} \mid y \in Y\right\}$ (otherwise $w \rightarrow y, w \rightarrow y^{\prime}$ would be unsatisfied); we write $W$ for $\left\{y, y^{\prime} \mid y \in Y\right\} \cup\{w\}$ - the set contained in each equilibrium model. Moreover, each equilibrium model $M$ must be of the form $V \cup\left\{z^{\prime} \mid z \in X \backslash V\right\} \cup W$. Indeed, one of $x$ and $x^{\prime}$ must hold for each $x \in X$ to satisfy $x \vee x^{\prime}$, but not both, as otherwise $\langle M \backslash\{x\}, M\rangle \models_{H T} P_{\phi}^{g}$ as well, contradicting the fact that $M$ is an equilibrium model of $P_{\phi}^{g}$. For each interpretation $I \subseteq X$ we write $I^{+}=I \cup\left\{x^{\prime} \mid x \in X \backslash I\right\} \cup W$ of $P_{\phi}^{g}$. One can show that $I$ is a model of $\forall Y \phi(X, Y)$ if and only if $I^{+}$is an equilibrium model of $P_{\phi}^{g}$. Indeed, if $I$ satisfies $\forall Y \phi(X, Y)$, then $\left\langle I^{+}, I^{+}\right\rangle \models_{H T} P_{\phi}^{g}$ and for all $J \subset I^{+}$it holds that $\left\langle J, I^{+}\right\rangle \nvdash_{H T} P_{\phi}^{g}$. On the other hand, if $I$ does not satisfy $\forall Y \phi(X, Y)$ then there exists $J \subseteq Y$ such that $I \cup J \not \models \phi(X, Y)$. It follows that $\left\langle I^{+} \backslash\left(\{y \mid y \notin J\} \cup\left\{y^{\prime} \mid y \in J\right\} \cup\{w\}\right), I^{+}\right\rangle \models_{H T} P_{\phi}^{g}$ (the key element of the argument here is that $\phi\left[\neg z / z^{\prime}\right]$ contains no occurrences of negation, as $\phi$ is in negation normal form and so, $I^{+} \backslash\left(\{y \mid y \notin J\} \cup\left\{y^{\prime} \mid y \in J\right\} \cup\{w\}\right) \not \forall \phi\left[\neg z / z^{\prime}\right]$; that property allows one to show $\left.\left.\left\langle I^{+} \backslash\left(\{y \mid y \notin J\} \cup\left\{y^{\prime} \mid y \in J\right\} \cup\{w\}\right), I^{+}\right\rangle\right|_{H T} \phi\left[\neg z / z^{\prime}\right] \rightarrow w\right)$. Thus, $I^{+}$is not an equilibrium model of $P_{\phi}^{g}$.

Once the correspondence between models $M$ of $\forall Y \phi(X, Y)$ and outcomes (equilibrium models) $M^{+}$of $P_{\phi}$ is established, we can handle the issue of minimality as in the case of CO problems. Let us assume first that $M^{+} \in \pi\left(P_{\phi}\right)$. Then, as we just demonstrated, $M$ satisfies $\forall Y \phi(X, Y)$. Moreover, we have that for any $N \subset M, N$ does not satisfy $\forall Y \phi(X, Y)$. Indeed if $N$ would satisfy $\forall Y \phi(X, Y)$, then we would have (i) $N^{+} \in \mu\left(P_{\phi}\right)$, (ii) for rules $r \in P_{\phi}^{s}$ of the form $u^{\prime}>u$, where $u \in M \backslash N, v_{N^{+}}(r)=1<2=v_{M^{+}}(r)$, and (iii) for all other rules $r^{\prime}$ in $P_{\phi}^{s}, v_{N^{+}}\left(r^{\prime}\right)=2=v_{M^{+}}\left(r^{\prime}\right)$; these three properties would imply $N^{+}>^{P_{\phi}} M^{+}$, contradicting $M^{+} \in \pi\left(P_{\phi}\right)$. Conversely, let us assume that $M$ is a minimal model of $\forall Y \phi(X, Y)$. Then $M^{+} \in \mu\left(P_{\phi}\right)$ and for all $N^{+} \in \mu\left(P_{\phi}\right)$ we can show that $N^{+}>^{P_{\phi}} M^{+}$does not hold 
(implying $M^{+} \in \pi\left(P_{\phi}\right)$ ). To this end, we reason as follows. If $N^{+}>^{P_{\phi}} M^{+}$would hold, then $v_{N^{+}}(r)<v_{M^{+}}(r)$ for at least one $r \in P_{\phi}^{s}$, and $v_{N^{+}}\left(r^{\prime}\right) \leq v_{M^{+}}\left(r^{\prime}\right)$, for all $r^{\prime} \in P_{\phi}^{s}$. This of course implies $N \subset M$ and, since $N$ satisfies $\forall Y \phi(X, Y)$ (we recall that $N^{+}$is an equilibrium model of $P_{\phi}^{g}$ and so, $N$ is a model of $\forall Y \phi(X, Y)$ ), it contradicts the assumption that $M$ is a minimal model of $\forall Y \phi(X, Y)$. We thus have that $M^{+} \in \pi\left(P_{\phi}\right)$ if and only if $M$ is a minimal model of $\forall Y \phi(X, Y)$. From that fact, reasoning as in the case of CO problems, we obtain that that $\forall Y \phi(X, Y)$ and $\forall Y \psi(X, Y)$ have the same minimal models if and only if $P_{\phi} \equiv{ }^{s, \geq 2} P_{\psi}$.

Theorem 23 Given two $C O$ (ASO, respectively) problems $P$ and $Q$, deciding $P \equiv_{g} Q$ is co-NP-complete.

Proof. Deciding strong equivalence of propositional theories under the classical semantics is co-NP-hard as in such case, strong equivalence and equivalence coincide. It is also coNP-hard under the equilibrium-model semantics (Lin, 2002). Thus, the hardness part of the assertion follows from Corollary 15.

To prove membership, we consider the complementary problem. By Theorem 13, it consists of deciding whether at least one of the conditions (1) and (2) below holds:

1. $P^{g}$ and $Q^{g}$ are not strongly equivalent or, equivalently, $\operatorname{Mod}\left(P^{g}\right) \neq \operatorname{Mod}\left(Q^{g}\right)$, for $\mathrm{CO}$ problems, and $\operatorname{Mod}_{H T}\left(P^{g}\right) \neq \operatorname{Mod}_{H T}\left(Q^{g}\right)$, for ASO problems;

2. $>_{\operatorname{Mod}\left(P^{g}\right)}^{P} \neq>_{\operatorname{Mod}\left(Q^{g}\right)}^{Q}$ or, equivalently, for some interpretations $I, J$ that are models of both $P^{g}$ and $Q^{g}$, exactly one of the properties $I>^{P} J$ and $I>^{Q} J$ holds.

Consequently, we consider a pair of interpretations $I, J$ to be a witness for an instance of that problem to be a YES instance if $I$ is a model (for CO problems) or $\langle I, J\rangle$ is an HT-model (for ASO problems) of exactly one of the two theories $P^{g}$ and $Q^{g}$, or if $I, J$ are models of both $P^{g}$ and $Q^{g}$ and exactly one of the properties $I>^{P} J$ and $I>^{Q} J$ holds.

It is easy to see that if a witness exists, then there is a witness $I, J$ such that $I$ and $J$ consist only of atoms that occur in $P$ and $Q$. After such a witness is guessed, verifying it (showing that condition (1) or (2) holds) can be done in polynomial time. It is well known that checking whether $I$ is a model $(\langle I, J\rangle$ is an HT-model) of a propositional theory can be done in polynomial time, and the same holds true for deciding $I>^{P} J$ and $I>^{Q} J$ (Lemma 33). Thus, the complementary problem is in NP.

Theorem 24 Given ranked $C O$ (ASO, respectively) problems $P$ and $Q$, and rank interval $[i, j]$, deciding $P \equiv_{g}^{s,[i, j]} Q$ is co-NP-complete.

Proof. For membership, we consider the complementary problem. By Theorem 16, it consists of deciding whether at least one of the conditions (1) - (4) below holds:

1. $P^{g}$ and $Q^{g}$ are not strongly equivalent (that is, $\operatorname{Mod}\left(P^{g}\right) \neq \operatorname{Mod}\left(Q^{g}\right)$ for CO problems, $\operatorname{Mod}_{H T}\left(P^{g}\right) \neq \operatorname{Mod}_{H T}\left(Q^{g}\right)$ for ASO problems);

2. for some interpretations $I, J$ that are models of both $\operatorname{Mod}\left(P^{g}\right)$ and $\operatorname{Mod}\left(Q^{g}\right)$, exactly one of properties $I>_{\operatorname{Mod}\left(P^{g}\right)}^{P} J$ and $I>_{\operatorname{Mod}\left(Q^{g}\right)}^{Q} J$ holds; 
3. for some $I, J \in \operatorname{Mod}\left(P^{g}\right)$ such that $i<\operatorname{diff}^{P}(I, J)$ or $i<\operatorname{diff}^{Q}(I, J)$, $\operatorname{diff}^{P}(I, J) \neq$ $\operatorname{diff}^{Q}(I, J)$ and it holds that $\operatorname{diff}^{P}(I, J) \leq j$ or $\operatorname{diff}^{Q}(I, J) \leq j$;

4. $>_{\operatorname{Mod}\left(P^{g}\right)}^{P_{<i}} \neq>_{\operatorname{Mod}\left(Q^{g}\right)}^{Q}$ or, equivalently, for some interpretations $I, J$ that are models of both $\operatorname{Mod}\left(P^{g}\right)$ and $\operatorname{Mod}\left(Q^{g}\right)$, exactly one of properties $I>^{P<i} J$ and $I>^{Q_{<i}} J$ holds.

We prove that the complementary problem is in NP. As in other proofs, we use for a membership witness a pair of interpretations $I, J$ that explicitly demonstrates that one of the conditions (1) - (4) holds. As before, if such a witness exists, there is also one consisting of atoms occurring in programs $P$ and $Q$ only. For each condition, given such a pair $I, J$ (restricted to atoms from $P$ and $Q$ ), one can verify in polynomial time whether the condition holds (for condition (1), we repeat the argument from the previous proof, for conditions (2) and (4), we use Lemma 33, and for condition (3) - Lemma 32).

Hardness follows directly from co-NP-completeness of deciding strong equivalence between two propositional theories under either of the semantics we consider and from Corollary 17 .

\section{References}

Boutilier, C., Brafman, R., Domshlak, C., Hoos, H., \& Poole, D. (2003). CP-nets: A tool for representing and reasoning with conditional ceteris paribus preference statements. Journal of Artificial Intelligence Research, 21, 135-191.

Boutilier, C., Brafman, R., Domshlak, C., Hoos, H., \& Poole, D. (2004). Preference-based constrained optimization with CP-nets. Computational Intelligence, 20, 137-157.

Brewka, G., Niemelä, I., \& Syrjänen, T. (2004). Logic programs with ordered disjunctions. Computational Intelligence, 20(2), 335-357.

Brewka, G., Niemelä, I., \& Truszczyński, M. (2003). Answer set optimization. In Gottlob, G., \& Walsh, T. (Eds.), Proceedings of the 18th International Joint Conference on Artificial Intelligence (IJCAI 2003), pp. 867-872. Morgan Kaufmann.

Brewka, G., Niemelä, I., \& Truszczyński, M. (2011). Answer set optimization. Unpublished manuscript.

Calimeri, F., Ianni, G., Krennwallner, T., \& Ricca, F. (2012). The answer set programming competition. AI Magazine, 33(4), 114-118.

Delgrande, J. P., Schaub, T., Tompits, H., \& Wang, K. (2004). A classification and survey of preference handling approaches in nonmonotonic reasoning. Computational Intelligence, 20(2), 308-334.

Eiter, T., Faber, W., Fink, M., \& Woltran, S. (2007a). Complexity results for answer set programming with bounded predicate arities and implications. Annals of Mathematics and Artificial Intelligence, 51 (2-4), 123-165.

Eiter, T., Fink, M., \& Woltran, S. (2007b). Semantical characterizations and complexity of equivalences in answer set programming. ACM Transactions on Computational Logic, $8(3)$. 
Eiter, T., \& Gottlob, G. (1995). On the computational cost of disjunctive logic programming: Propositional case. Annals of Mathematics and Artificial Intelligence, 15(3/4), 289323.

Faber, W., Truszczyński, M., \& Woltran, S. (2012). Strong equivalence of qualitative optimization problems. In Brewka, G., Eiter, T., \& McIlraith, S. A. (Eds.), Proceedings of the 13th International Conference on Principles of Knowledge Representation and Reasoning (KR 2012), pp. 188-198. AAAI Press.

Faber, W., \& Konczak, K. (2006). Strong order equivalence. Annals of Mathematics and Artificial Intelligence, 47(1-2), 43-78.

Faber, W., Tompits, H., \& Woltran, S. (2008). Notions of strong equivalence for logic programs with ordered disjunction. In Brewka, G., \& Lang, J. (Eds.), Proceedings of the 11th International Conference on Principles of Knowledge Representation and Reasoning (KR 2008), pp. 433-443. AAAI Press.

Ferraris, P. (2005). Answer sets for propositional theories. In Baral, C., Greco, G., Leone, N., \& Terracina, G. (Eds.), Proceedings of the 8th International Conference on Logic Programming and Nonmonotonic Reasoning (LPNMR 2005), Vol. 3662 of Lecture Notes in Computer Science, pp. 119-131. Springer.

Ferraris, P., \& Lifschitz, V. (2005). Mathematical foundations of answer set programming. In We Will Show Them! Essays in Honour of Dov Gabbay, pp. 615-664. College Publications.

Gelfond, M., \& Lifschitz, V. (1991). Classical negation in logic programs and disjunctive databases. New Generation Computing, 9, 365-385.

Goldsmith, J., Lang, J., Truszczyński, M., \& Wilson, N. (2008). The computational complexity of dominance and consistency in CP-nets. Journal of Artificial Intelligence Research, 33, 403-432.

Goldsmith, J., \& Junker, U. (Eds.). (2008). Special Issue on Preferences, Vol. 29(4) of AI Magazine.

Heyting, A. (1930). Die formalen Regeln der intuitionistischen Logik. Sitzungsberichte der Preussischen Akademie der Wissenschaften, 1, 42-56.

Kaci, S. (2011). Working with Preferences. Springer.

Lifschitz, V. (1985). Computing circumscription. In Joshi, A. K. (Ed.), Proceedings of the 9th International Joint Conference on Artificial Intelligence (IJCAI 1985), pp. 121-127. Morgan Kaufmann.

Lifschitz, V., Pearce, D., \& Valverde, A. (2001). Strongly equivalent logic programs. ACM Transactions on Computational Logic, 2(4), 526-541.

Lin, F. (2002). Reducing strong equivalence of logic programs to entailment in classical propositional logic. In Fensel, D., Giunchiglia, F., McGuiness, D. L., \& Williams, M.A. (Eds.), Proceedings of the 8th International Conference on Principles of Knowledge Representation and Reasoning (KR 2002), pp. 170-176. Morgan Kaufmann.

Marek, V. W., \& Truszczyński, M. (1993). Nonmonotonic Logic; Context-Dependent Reasoning. Springer, Berlin. 
Marek, V., \& Truszczyński, M. (1999). Stable models and an alternative logic programming paradigm. In Apt, K., Marek, W., Truszczyński, M., \& Warren, D. (Eds.), The Logic Programming Paradigm: a 25-Year Perspective, pp. 375-398. Springer, Berlin.

Niemelä, I. (1999). Logic programming with stable model semantics as a constraint programming paradigm. Annals of Mathematics and Artificial Intelligence, 25(3-4), 241-273.

Pearce, D. (1997). A new logical characterisation of stable models and answer sets. In Dix, J., Pereira, L. M., \& Przymusinski, T. (Eds.), Proceedings of the 6th International Workshop on Non-Monotonic Extensions of Logic Programming (NMELP 1996), Vol. 1216 of Lecture Notes in Computer Science, pp. 57-70. Springer.

Pearce, D., Tompits, H., \& Woltran, S. (2009). Characterising equilibrium logic and nested logic programs: Reductions and complexity. Theory and Practice of Logic Programming, 9(5), 565-616.

Simons, P., Niemelä, I., \& Soininen, T. (2002). Extending and implementing the stable model semantics. Artificial Intelligence, 138, 181-234.

Turner, H. (2003). Strong equivalence made easy: Nested expressions and weight constraints. Theory and Practice of Logic Programming, 3(4-5), 609-622. 